\title{
Pathophysiologie des Schocks
}

K. Werdan, U. Müller-Werdan

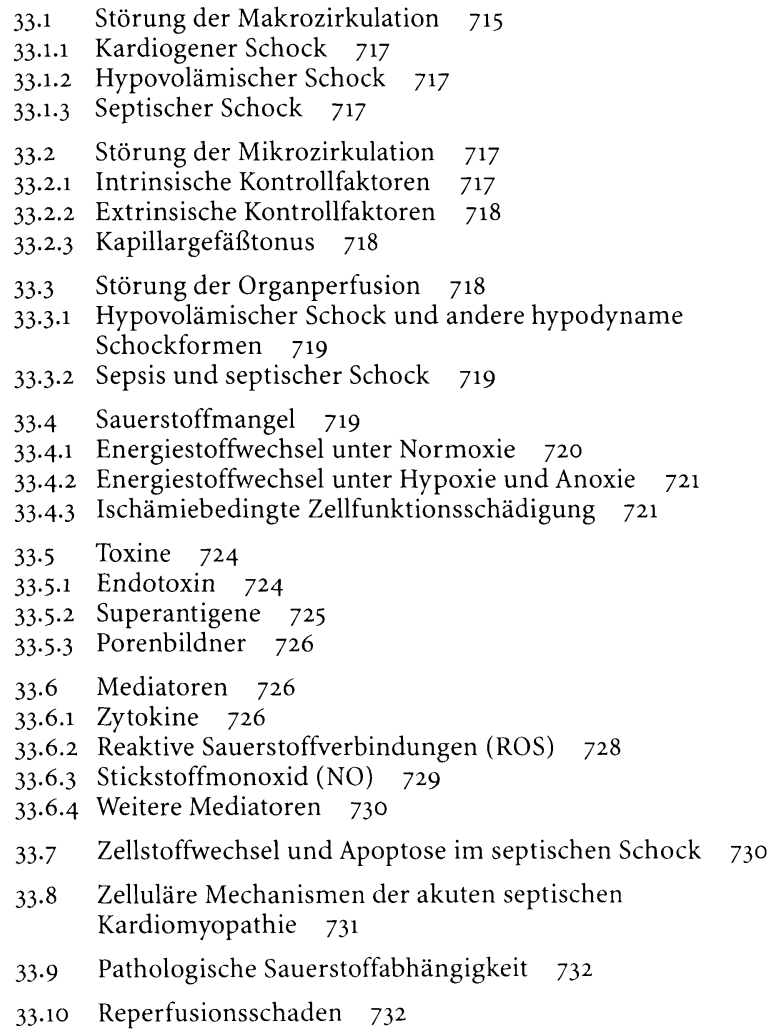

33.11 Neurohumorale und para-/autokine Adaptation 733

33.11.1 Aufrechterhaltung des venösen Zirkulationsdrucks 733

33.11.2 Optimierung der Herzfunktion 735

33.11.3 Blutumverteilung 735

33.11.4 Erleichterung der Sauerstoffabgabe im Gewebe 735

Abbildung 33-1 verdeutlicht die Komplexität des Schockgeschehens. Die folgende auszugsweise Besprechung einzelner Schockkomponenten kann demzufolge immer nur einen Teilaspekt beleuchten.

\section{1}

\section{Störung der Makrozirkulation}

Die Betrachtung der hämodynamischen Schockauswirkungen muß neben dem arteriellen Schenkel und der Mikrozirkulation auch den venösen Schenkel des Kreislaufsystems miteinbeziehen (Abb. 33-2a-c).

Der venöse Rückstrom zum Herzen ist proportional dem Quotienten

$$
\left(\mathrm{pcm}-\mathrm{p}_{\mathrm{RA}}\right) / \mathrm{Rv}
$$

(pcm mittlerer Zirkulationsdruck (intravasaler Venendruck bei ruhendem Herzen); $p_{\text {RA }}$ rechtsatrialer Mitteldruck; Rv venöser Gefäßwiderstand,
Abb. 33-1. Schock - Pathogenese und Pathophysiologie

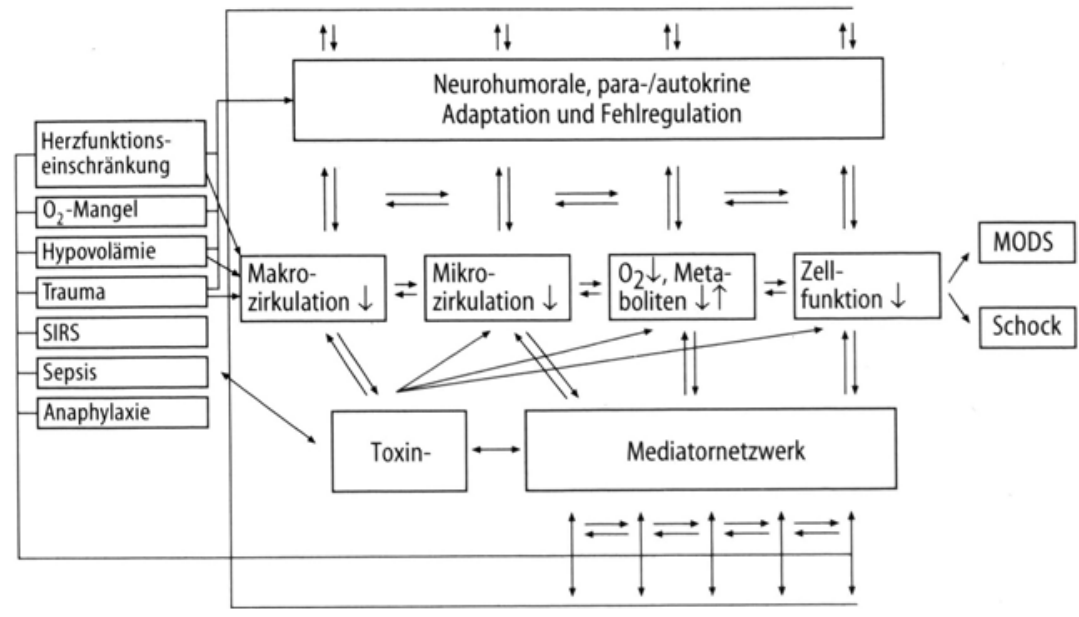


Abb. 33-2a-c. Darstellung der Interaktion von venösem Rückstrom und rechtsventrikulärer Herzfunktion bei kardiogenem Schock (a), hypovolämischem Schock (b) und septischem Schock (c). $p_{R A}$ rechtsatrialer Mitteldruck im rechten Vorhof; pcm mittlerer venöser Zirkulationsdruck; $R v$ venöser Gefäßwiderstand; $V_{t}$ Gesamtblutvolumen. Therapeutische Maßnahmen, deren Wirkungen und weitere Erläuterungen s. Text

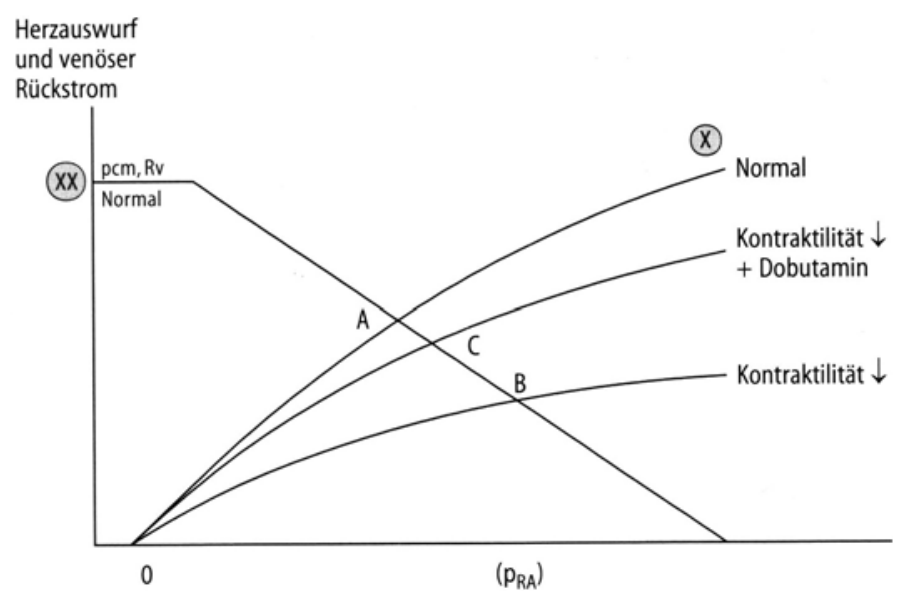

b
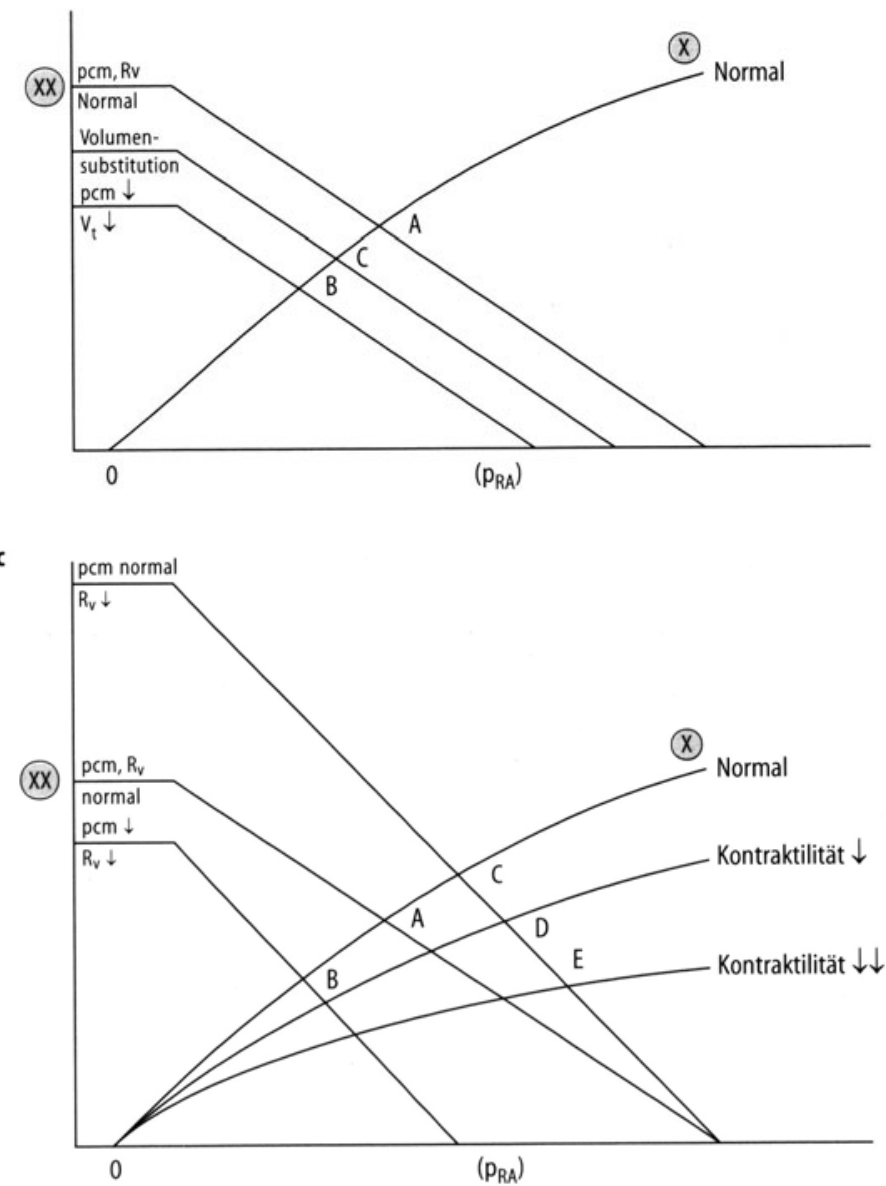

pcm entspricht dabei dem Quotienten aus Vs/C, Vs Blutvolumen, das den Venendruck bestimmt; es errechnet sich aus der I)ifferenz des gesamten Blutvolumens Vt und dem Blutvolumen Vo (Blutvolumen bei Atmosphärendruck, d.h. nach passiver Exsanguination verbleibendes Blutvolumen); (' Compliance.)
In einem geschlossenen Kreislaufsystem ist der Herzauswurf (Herzzeitvolumen, determiniert durch Herzfrequenz, Vorlast, Nachlast und Kontraktilität) mit dem venösen Rückstrom (venöses Minutenvolumen, determiniert durch mittleren Zirkulationsdruck, rechtsatrialen Druck und venösen Gefäßßwiderstand) identisch. Dem- 
zufolge lassen sich die rechtsventrikuläre Frank-Starling-Kurve $(\mathrm{x})$ und die venöse Rückstromkurve ( $\mathrm{xx}$ ) graphisch kombinieren (Abb. 33-2a-c). Der venöse Rückstrom korreliert linear mit dem rechtsatrialen Druck $\mathrm{p}_{\mathrm{RA}}$ bis $\mathrm{p}=\mathrm{o} \mathrm{mmHg}$ (= Atmosphärendruck), bei dem die großen Venen intermittierend kollabieren und damit den venösen Rückstrom limitieren und das gezeigte Plateau produzieren.

Die Steigung der venösen Rückstromkurve ist der Reziprokwert des venösen Gefäßwiderstandes ( $1 / \mathrm{Rv}$ ). Der Schnittpunkt der venösen Rückstromkurve mit der x-Achse repräsentiert dabei den mittleren Zirkulationsdruck pcm; Veränderungen des $\mathrm{pcm}$ verschieben dabei die venöse Rückstromkurve nach links bzw. nach rechts ohne Änderung der Steigung; Änderungen des venösen Widerstandes Rv ändern die Steigung der Kurve, eine Zunahme von Rv führt zu einer Abflachung, eine Abnahme zu einer größeren Steilheit der Kurve (ausführliche Diskussion in Kumar u. Parrillo 1995a).

\subsection{1}

\section{Kardiogener Schock}

Der Verlust von Myokardmasse beim akuten Herzinfarkt mit Minderung der Kontraktilität führt zur Abflachung der Frank-Starling-Kurve und zur Zunahme des Vorhofdrucks (Abb. 33-2a, A-B). Durch das positiv-inotrop wirkende Dobutamin (ohne wesentlichen Einfluß auf den venösen Rückfluß) kann die Kontraktionskraft des Herzens gesteigert und damit der Herzauswurf erhöht werden (Abb. 33-2a, B-C). Im Gegensatz zum Dobutamin würden die Katecholamine Noradrenalin und Dopamin nicht nur positiv-inotrop wirken, sondern auch die venöse Kapazität reduzieren und damit den mittleren Zirkulationsdruck erhöhen.

\subsection{2}

\section{Hypovolämischer Schock}

Hypovolämie vermindert das zirkulierende Blutvolumen und damit auch den mittleren Zirkulationsdruck pcm (Abb. 33-2b, A-B). Die resultierende Abnahme des venösen Rückstroms und damit des Herzauswurfs kann durch Volumensubstitution ausgeglichen werden (Abb. 33-2b, B-C).

Das hämodynamische Bild des hämorrhagischen hypovolämischen Schocks ist nicht nur durch den Blutvolumenverlust geprägt, sondern auch durch eine initiale kompensatorische Vasokonstriktion, welche im weiteren Verlauf von einer therapeutisch schwer beeinflußbaren Vasodilatation gefolgt wird. Für letztere wird zum einen eine Hochregulation der spinalen Stickoxidsynthase verantwortlich gemacht (Lu et al. 1999), zum anderen eine Aktivierung der Poly-(ADP-Ribose)-Synthetase (PARS), einem Schlüsselenzym der Apoptose (s. Abschn. 33.7).

\subsection{3 \\ Septischer Schock}

Der septische Schock beeinflußt das venöse System in zweierlei Hinsicht: einerseits führen die aktive Dilatation kleiner Venen und Venolen sowie der Plasmaabfluß ins Interstitium zur Steigerung der Venenkapazität bei gleichzeitiger Abnahme des Blutvolumens; eine Abnahme des pcm und damit des venösen Rückstroms und konsekutiv des Herzauswurfs ist die Folge. Andererseits findet sich im septischen Schock auch eine Dilatation der großen Venen und ein arteriovenöses Shunting, beides mit der Folge einer Verminderung des venösen Widerstandes (Rv) und damit einem verstärkten venösen Rückstrom zum Herzen.

Vor adäquater Volumensubstitution reicht die Abnahme des Rv allerdings nicht aus, um die Reduktion des pcm auszugleichen: Der Herzauswurf bleibt vermindert (Abb. 33-2c, A-B). Durch eine adäquate Volumensubstitution läßt sich der pcm normalisieren; aufgrund des weiterhin erniedrigten venösen Widerstands (Rv) führt dies, bei Fehlen einer Myokarddepression, dann sogar zu einem supranormalen venösen Rückstrom und einem supranormalen Herzzeitvolumen (Abb. 33-2c, B-C). Die Graphik verdeutlicht, daß die Rv-Abnahme bis zu einem gewissen Grad eine meist vorhandene mäßige Myokarddepression maskieren kann. Erst bei einer ausgeprägten Kontraktilitätsminderung wird diese als Einschränkung des Herzauswurfs manifest (Abb. 33-2c, C-D), was bei ca. $20 \%$ aller Sepsispatienten zutrifft (Kumar u. Parrillo 1995a).

\section{2}

\section{Störung der Mikrozirkulation}

Eine regelrechte Durchblutung auf Mikrozirkulationsebene (Gefäße bis 100-150 $\mu \mathrm{m}$ Durchmesser) ist Voraussetzung für eine adäquate Gewebeperfusion; ein normales Herzzeitvolumen und ein normaler Blutdruck sind dafür noch nicht ausreichend.

Die regionale Durchblutung auf Mikrozirkulationsebene wird durch lokale intrinsische (Autoregulation) und durch extrinsische Faktoren (autonomes Nervensystem und humorale Faktoren) geregelt. Der Blutfluß zu den einzelnen Organen wird durch den Tonus der präkapillären Arteriolen und prä- und postkapillären Sphinktergefäße sowie durch lokale Veränderungen der metabolischen Aktivität gesteuert.

\subsection{1}

\section{Intrinsische Kontrollfaktoren}

Intrinsische Kontrollfaktoren (Autoregulation) des Blutflusses sind endotheliale Dehnungsrezeptoren: plötzliche Änderungen des Perfusionsdrucks können so mit entgegengesetzten Änderungen des Gefäßswiderstands gegenreguliert werden, um die Durchblutung aufrecht- 
zuerhalten (Johnson 1986). Eine Durchblutungssteigerung zur Deckung erhöhter metabolischer Aktivitäten kann über die Freisetzung verschiedener Metabolite (z.B. $\mathrm{CO}_{2}, \mathrm{H}^{+}$) mit vasodilatierender Wirkung erzielt werden.

\subsection{2}

\section{Extrinsische Kontrollfaktoren}

Die extrinsische Kontrolle des Vasomotorentonus wird im wesentlichen über das autonome Nervensystem erzielt. Die Freisetzung von Acetylcholin über Parasympathikusfasern führt in Endothel- und Gefäßmuskelzellen über die Bildung von Stickoxid und zyklischem Guanosinmonophosphat zur Vasodilatation. Eine Steigerung des Sympathikotonus führt zur lokalen Noradrenalinfreisetzung und damit, über eine Besetzung vaskulärer $\alpha$-Adrenozeptoren, zur Vasokonstriktion. Streß bewirkt über eine Sympathikusaktivierung die Freisetzung von Adrenalin und Noradrenalin aus dem Nebennierenmark. Das Renin-Angiotensin-System kontrolliert Blutdruck und -fluß unter Basalbedingungen.

\subsection{3}

\section{Kapillargefäßtonus}

Die Mikrozirkulation wird durch prä- und postkapilläre Gefäßregionen kontrolliert, die beide auf intrinsische und extrinsische Kontrollmechanismen ansprechen. Änderungen des Tonus dieser Gefäßregionen sind insofern bedeutungsvoll, als zwischen diesen beiden Gefäßregionen (auf der Kapillarebene) ein aktiver Stoffwechsel und die Flüssigkeitsregulation über die Kapillarwand stattfindet. Das Öffnen von mikroanatomischen oder funktionellen Shunts führt zu einem Mismatch von Stoffwechsel und $\mathrm{O}_{2}$-Versorgung; das Nichtöffnen von Gefäßsphinktern metabolisch aktiver Regionen führt zu Ischämie und zum anaeroben Stoffwechsel mit Laktatproduktion.

Ein erhöhter präkapillärer Tonus (wie bei Sympathikusstimulation) führt zur Erhöhung des systemischen Blutdrucks und zur Senkung des lokalen hydrostatischen Drucks. Der erniedrigte hydrostatische Druck favorisiert die Volumenumverteilung aus dem Interstitium zurück in den Kreislauf. Ein relativ zum präkapillären Tonus erhöhter postkapillärer Tonus führt zum Gefäßpooling von Blut und zum Verlust von Flüssigkeit ins Interstitium infolge des erhöhten hydrostatischen Drucks. I)ie Störung der Mikrozirkulation ist ein wesentliches Charakteristikum des Schocks. Sie beruht zum einen auf dem Verlust der Vasomotorenregulation mit daraus resultierendem Mismatch von Gewebedurchblutung und metabolischer Aktivität; zum anderen trägt auch der Verlust der Endothelintegrität mit seinen Folgen dazu bei, ebenso wie das intravasale "clotting“ von Blutellen und die verminderte Erythrozytendeformierbarkeit. 1)ie diesen störungen zugrundeliegenden, bei den einzelnen Schockformen qualitativ und quantitativ unterschiedlich relevanten Mechanismen sind in der folgenden Übersicht aufgeführt.

\section{Vasomotorenstörung und Mikrozirkulationsschädigung im Schock}

Verlust der Vasomotorenregulation mit Mismatch von Gewebedurchblutung und metabolischer Aktivität durch (Thijs u. Groenefeld 1988):

- Gewebeazidose;

- Gewebekatecholaminverarmung;

- mediatorinduzierte Katecholaminresistenz der Gefäßmuskulatur;

- Freisetzung von vasodilatierenden und vasokonstringierenden Prostaglandinmetaboliten;

- verminderten Sympathikotonus infolge zerebraler Durchblutungsstörungen;

- mediatorinduzierter Bildung von Stickoxid in Gefäßmuskelzellen.

- Verlust der Endothelintegrität durch:

- reaktive $\mathrm{O}_{2}$-Verbindungen, gebildet von aktivierten neutrophilen Granulozyten und während der Reperfusion;

- Freisetzung vasoaktiver Faktoren wie Histamin, Bradykinin, plättchenaktivierendem Faktor, Leukotriene, Tumornekrosefaktor;

- Leukozyten-Endothel-Interaktion via Adhäsionsmoleküle (Integrine, Selectine).

Folgen des Verlustes der Endothelintegrität:

- erhöhte Kapillarpermeabilität;

- Exsudation von Plasmaproteinen ins Gewebe;

- Abfall des plasmaonkotischen Drucks;

- Entwicklung eines interstitiellen Ödems;

- Abnahme des zirkulierenden Blutvolumens.

Intravasales "clotting" von Erythrozyten, Leukozyten und Thrombozyten mit Verstärkung der Endothelschädigung und Verschlimmerung des Mismatches durch (Thijs und Groenefeld 1988):

- Mikrothromben;

- primäre Endothelschädigung durch zirkulierende Zytokine;

- reaktive $\mathrm{O}_{2}$-Verbindungen, generiert durch neutrophile Granulozyten und während der Reperfusion;

- Komplementaktivierung.

Verminderte Erythrozytendeformierbarkeit infolge durch reaktive $\mathrm{O}_{2}$-Verbindungen hervorgerufener Membranschäden.

\section{3}

\section{Störung der Organperfusion}

Die Durchblutung der einzelnen Organe kann über einen weiten Blutdruckbereich organspezifisch konstant gehalten werden (Tabelle 33-1), für den Menschen wird diese Autoregulation für einen Bereich von $60-100 \mathrm{mmHg}$ angegeben (Bond 1993).

Außerhalb dieses Bereichs müssen extrinsische Adaptationsmechanismen zum Tragen kommen, soll es nicht durch ein Mismatch von Durchblutung und metabolischer Aktivität zum Organversagen und Schock kommen. 
Tabelle 33-1. Organspezifische Gefäßleitfähigkeit bei Normotonie und bei lokaler, durch Exsanguination oder durch Endotoxininfusion ausgelöster Hypotonie in Hunden. Die Angaben zur Autoregulation beziehen sich auf Messungen mit lokaler Blutdrucksenkung im entsprechenden Organgefäßbett. n.a. nicht angegeben. (Mod. nach Bond 1993)

\begin{tabular}{|c|c|c|c|c|c|c|}
\hline \multirow[t]{3}{*}{ Organperfusion } & \multirow[t]{3}{*}{ Herzzeitvolumen } & \multirow{3}{*}{$\begin{array}{l}\text { Autoregulation } \\
\text { [mmHg] }\end{array}$} & \multicolumn{4}{|c|}{ Gefäßleitfähigkeit [ml/mmHg/min] } \\
\hline & & & \multirow{2}{*}{$\begin{array}{l}\text { Kontrolle } \\
{[100 \mathrm{mmHg}]}\end{array}$} & \multicolumn{3}{|c|}{ Hypotension [60 mmHg] } \\
\hline & & & & Lokal & Hämorrhagie & Endotoxin \\
\hline Gehirn & $3,6 \%$ & $30-200$ & $1,08(100 \%)$ & $1,51(140 \%)$ & $1,46(135 \%)$ & $0,59(55 \%)$ \\
\hline Herz & $4,7 \%$ & $40-100$ & $1,41(100 \%)$ & $2,05(145 \%)$ & $1,99(141 \%)$ & $2,12(150 \%)$ \\
\hline Mesenterialkreislauf & $25,1 \%$ & $>60$ & $7,53(100 \%)$ & $9,26(123 \%)$ & $4,52(60 \%)$ & $15,01(199 \%)$ \\
\hline Nieren & $25,4 \%$ & $>60$ & $7,62(100 \%)$ & $7,62(100 \%)$ & $3,05(40 \%)$ & $14,48(190 \%)$ \\
\hline Skelettmuskel & $28,2 \%$ & $50-100$ & $8,46(100 \%)$ & $10,99(130 \%)$ & $5,25(62 \%)$ & $11,67(138 \%)$ \\
\hline Haut & $12,9 \%$ & n.a. & $3,87(100 \%)$ & $3,10(80 \%)$ & $1,43(37 \%)$ & $4,64(120 \%)$ \\
\hline Gesamt & $99,9 \%$ & n.a. & $29,97(100 \%)$ & $34,53(115 \%)$ & $17,70(59 \%)$ & $48,51(162 \%)$ \\
\hline
\end{tabular}

\subsection{1}

\section{Hypovolämischer Schock und andere hypodyname Schock- formen}

Bei Hypovolämie und anderen hypodynamen Schockformen wird der Blutfluß in Gehirn und Herz autoregulatorisch aufrechterhalten, in allen anderen Organgefäßbetten ist er jedoch teilweise erheblich eingeschränkt, damit der systemische Blutdruck möglichst stabil gehalten werden kann (Tabelle 33-1). Für diese Vasokonstriktion sind v.a. ein erhöhter Sympathikotonus und die Freisetzung von Katecholaminen aus der Nebenniere (Baker et al. 1988) verantwortlich.

Diese adaptativen Mechanismen reichen aus, um bei gering bis mäßig eingeschränktem Herzzeitvolumen lebenswichtige Organe adäquat zu perfundieren; bei ausgeprägter Hypotonie kommt es jedoch zur Organischämie und zum Organversagen. Selbst nach Wiederherstellung stabiler Herz-Kreislauf-Verhältnisse können die Störungen auf Mikrozirkulationsebene über Tage persistieren, v.a. im Gehirn, in den Nieren, in der Leber und in anderen Splanchnikusorganen (Wang et al. 1990). Die irreversible Phase eines schweren hämorrhagischen Schocks ist aufgrund experimenteller Befunde durch die Vasodilatation präkapillärer Sphinkter charakterisiert (Bond u. Johnson 1985).

\subsection{2}

\section{Sepsis und septischer Schock}

Bei Sepsis und septischem Schock finden sich bereits bei noch relativ adäquaten Blutdruckwerten Störungen der Organdurchblutung, die eine primäre Schädigung auf Mikrozirkulationsebene nahelegen. Mit Ausnahme der Hirnstrombahn zeigen alle anderen Strombahngebiete (Splanchnikusbereiche, Niere, Skelettmuskel, Haut ) eine starke Abnahme des Gefäliswiderstandes (Tabelle 33-1).
Sie beruht auf einer sepsisinduzierten aktiven Vasodilatation und dem Verlust der extrinsischen Vasomotorenkontrolle (Bond 1993), wodurch die Durchblutung ausschließlich vom Herzzeitvolumen abhängig wird.

Eine Ausnahme von dieser Regel stellt die Hirndurchblutung dar, die in der Sepsis weiterhin die Fähigkeit zur Autoregulation beibehält: Bei Patienten mit Sepsis ist die Hirndurchblutung bereits vor der Ausbildung des Schockzustandes um $1 / 3$ reduziert (Bowton et al. 1989), wobei diese Durchblutungseinschränkung jedoch nicht als Ursache der septischen Enzephalopathie angesehen wird. Im Koronargefäßsystem fällt dagegen der Widerstand noch stärker ab als in den anderen Organen, und demzufolge ist die Koronarperfusion bei Patienten mit septischem Schock sogar häufig erhöht.

\section{4}

\section{Sauerstoffmangel}

Die Schädigung der Zellfunktion kommt durch ein komplexes Wechselspiel verschiedener auslösender Noxen und gehemmter/geschädigter Zellfunktionen zustande (Abb. 33-1). Den einzelnen Komponenten kommt je nach Schockform unterschiedliche Relevanz zu. Zentrale Bedeutung haben dabei der $\mathrm{O}_{2}$-Mangel, Toxine und Mediatoren sowie reaktive $\mathrm{O}_{2}$-Verbindungen. Letztere entfalten ihre schädliche Wirkung auch in der Reperfusionsphase nach adäquater initialer Schockbehandlung.

Zu den geschädigten Zellfunktionen zählen: der Energiestoffwechsel, Zellmembranfunktionen, Zellkernprozesse einschließlich Genexpression und Apoptose, Zellmetabolismus und (im Falle der Herzmuskelzelle) die Beeinträchtigung inotroper Mechanismen. Letztere können, wie beim septischen Schock, das Schockgeschehen mit auslösen und im weiteren Verlauf auch perpetuieren. Die folgende Diskussion (Werdan 1995a) konzentriert sich auf die schockbedingte Schädigung der Herzmuskelzelle. 


\subsection{1}

\section{Energiestoffwechsel unter Normoxie}

Etwa $60-70 \%$ des im Herzen gebildeten Adenosintriphosphats (ATP) werden bei der Kontraktion aufgebraucht (Abb. 33-3), einschließlich der dazu notwendigen Ionentransporte wie $\mathrm{Ca}^{2+}$-Aufnahme in das sarkoplasmatische Retikulum und $\mathrm{Na}^{+} / \mathrm{K}^{+}$-ATPase getriebener, aktiver $\mathrm{Na}^{+} / \mathrm{K}^{+}$-Transport über die Zellmembran (ca. 10-25\%). Nur relativ wenig ATP (weniger als 5\%) wird für die Generierung des Aktionspotentials und die Erregungsleitung des Herzens benötigt, noch weniger für die proteinkinasenvermittelte Phosphorylierung von Zellproteinen und für die Bildung des zyklischen Adenosinmonophosphats (cAMP), welches im Kardiomyozyten regulatorischen Schlüsselfunktionen im Kontraktionsablauf besitzt.

Geringe Mengen an ATP werden zur Aufrechterhaltung der Mitochondrienstruktur und deren Volumenregulation benötigt, weiterhin für die Synthese von Glykogen, Triglyzeriden und Protein und als zellmembranassoziierter ATP-Speicher. Da der Proteinstoffwechsel im Herzen eine hohe Umsatzrate aufweist, muß auch laufend ATP zur Proteinsysnthese bereitgestellt werden: Eine ATP-Verarmung von $40 \%$ führt in neonatalen Rattenkardiomyozyten zu einer Hemmung der Proteinsynthese um 35\%. Der Effektivitätsgrad der Umsetzung von chemischer Energie (ATP) in mechanische Arbeit beträgt im Herzen ca. 20-15\%.

Die wesentlichen Funktionen des Kreatinphosphats (KP) im Herzen sind die Wiederauffüllung von ATPPools und der "Energietransport" durch das Zytosol. Darüber hinaus fungiert es auch als Speicher für anorganisches Phosphat $\left(\mathrm{P}_{\mathrm{i}}\right)$, das beim Zerfall von ATP freigesetzt wird. Der rasche Anstieg von $\mathrm{P}_{\mathrm{i}}$ im Zytosol zeigt eine vermehrte Herzarbeit oder $\mathrm{O}_{2}$-Mangel an, $\mathrm{P}_{\mathrm{i}}$ stimuliert die Glykolyse, indem es die Aktivität des Schlüsselenzyms Phosphofruktokinase steigert; $\mathrm{P}_{\mathrm{i}}$ steigert als Substrat der Phosphorylase die Glykogenolyse und stimuliert auch über einen noch nicht aufgeklärten Mecha- nismus die oxidative Phosphorylierung. Die übermäßige Anhäufung von $\mathrm{P}_{\mathrm{i}}$ im Gefolge einer Ischämie kann paradoxerweise die Kontraktilität hemmen und damit den $\mathrm{O}_{2}$-Verbrauch reduzieren.

Innerhalb der Zelle bestehen 2 ATP-Kompartimente: die Mitochondrien als Ort seiner überwiegenden Synthese und das Zytosol als Ort des überwiegenden Verbrauchs. Mehr als 90\% des ATP sind dabei im Zytosol zu finden. Während der Herzarbeit wird zytosolisches ATP verbraucht. Dadurch kommt es zu einer Verdopplung der sehr niedrig gehaltenen zytosolischen Adenosindiphosphat(ADP)-Konzentration.

Dieser ADP-Anstieg stimuliert wiederum die mitochondriale ATP-Produktion via oxidative Phosphorylierung. Das Konzept der Subkompartimentierung des ATP (Abb. 33-4) innerhalb des Zytosols beinhaltet neben kleinen ATP-Speichern in der Nähe des $\mathrm{K}^{+}$-Kanals (ATP blockiert den $\mathrm{K}^{+}$-Flux) und auf der Zellmembranoberfläche (ATP scheint als Vasodilatator zu wirken) eine lokale Konzentrierung um die Myofibrillen und das sarkoplasmatische Retikulum (energieabhängige Aufnahme von $\left.\mathrm{Ca}^{2+}\right)$.

Die mitochondriale Kreatinphosphokinase ist auf der Außenseite der inneren Mitochondrienmembran lokalisiert: Sie transferiert die chemische Erergie des in den Mitochondrien gebildeten und mittels des Adeninnukleotidtranslokators (innere Mitochondrienmembran) in den Intermembranraum transportierten ATP auf das Kreatinphosphat. Da die äußere Mitochondrienmembran für KP frei permeabel ist, kann dieses in das Zytoplasma diffundieren, wo zytoplasmatische KP-Kinaseisoenzyme die KP-gespeicherte Energie auf ADP zur Bildung von ATP rückübertragen.

Die gemeinsame Endstrecke der Substratutilisation zur Energieproduktion wird durch den Krebs-Zyklus (Zitratzyklus, Trikarboxylatzyklus) und die Atmungskette repräsentiert, die beide in den Mitochondrien lokalisiert sind. Das gemeinsame Endprodukt der Substratutilisation unter aeroben Bedingungen ist das Acetyl-CoA, das aus Fetten über die $\beta$-Oxidation aktivierter Fettsäu-
Abb. 33-3. Funktion von Adenosintriphosphat $(A T P)$ und Kreatinphosphat $(K P)$ am Beispiel des Herzens. $P_{i}$ anorganisches Phosphat. (Mod. nach Werdan 1995a)

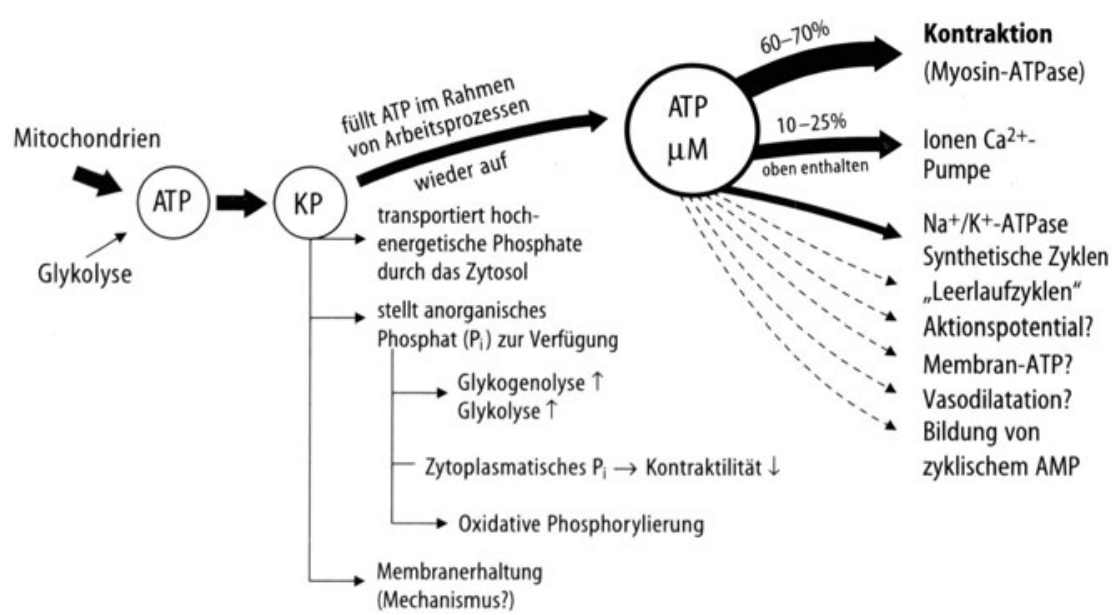


Abb.33-4. Kompartimentierung und Transfer energiereicher Phosphate in Kardiomyozyten. KP Kreatinphosphat $C K$ Kreatinphosphokinase; $S R$ sarkoplasmatisches Retikulum. (Mod. nach Werdan 1995a)

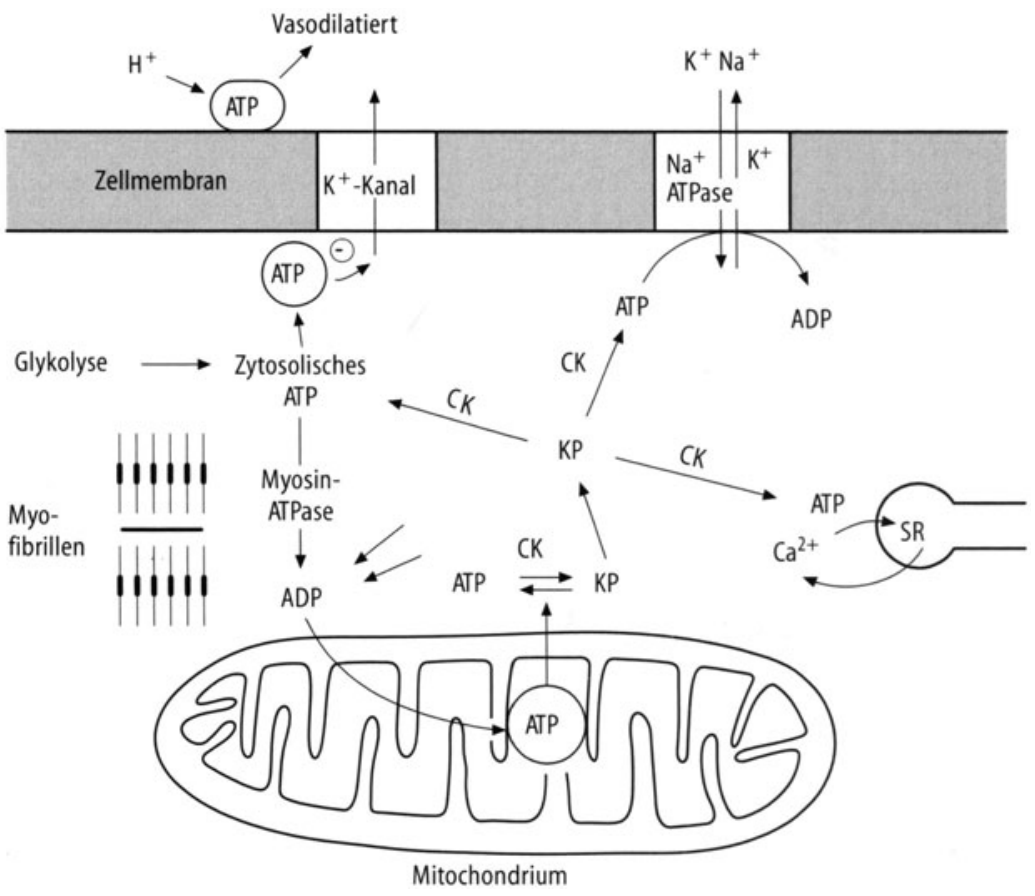

ren (Acyl-CoA), aus Glukose über die oxidative Dekarboxylierung des Pyruvats mittels der Pyruvatdehydrogenase und aus Ketokörpern mittels der Aktivierung der CoA gebildet wird.

Die Aktivität der Atmungskette wird durch zahlreiche Faktoren reguliert, wobei dem Sauerstoff aufgrund der sehr hohen $\mathrm{O}_{2}$-Affinität der Atmungskette (Michaelis-Konstante $<1 \mu \mathrm{M}$ ) nur unter den Bedingungen der schwersten Hypoxie eine regulierende Rolle zukommt: Unter normoxischen Bedingungen sind die ATP-Spiegel und der $\mathrm{O}_{2}$-Partialdruck in der Zelle hoch und die ADP-Spiegel niedrig stabil. Unter diesen Bedingungen scheint $\mathrm{NADH}+\mathrm{H}^{+}$die Geschwindigkeit der mitochondrialen ATP-Produktion zu bestimmen, da der $\mathrm{NAD}^{+}$/ $\mathrm{NADH}+\mathrm{H}^{+}$-Quotient relativ hoch ist.

\subsection{2}

\section{Energiestoffwechsel unter Hypoxie und Anoxie}

Bei Hypoxie oder milder Ischämie akkumulieren ADP und $\mathrm{NADH}+\mathrm{H}^{+}$. Unter diesen Bedingungen scheint der erhöhte zelluläre ADP-Spiegel die treibende Kraft für die mitochondriale ATP-Produktion darzustellen. Bei schwerer Ischämie oder Koronarverschluß akkumulieren $\mathrm{NADH}+\mathrm{H}^{+}$und $\mathrm{FADH}_{2}$ und kommen damit als Regulatoren nicht mehr in Frage. Auch das angestiegene ADP bestimmt unter diesen Bedingungen nicht mehr die Geschwindigkeit der ATP-Produktion, weil nun der sehr niedrige $\mathrm{O}_{2}$-Partialdruck zum geschwindigkeitsbestimmenden Schritt wird. Da die mitochondrialen $\mathrm{O}_{2-}^{-}$ Reserven nur sehr klein sind, stirbt der Kardiomyozyt nach Erreichen dieses Zustands rasch ab.
Bei Hypoxie und experimenteller Anoxie ist die Atmungskette gehemmt. Bei einer vollständigen Blockade der Atmungskette kann die ATP-Produktion nur noch via anaerobe Glykolyse erfolgen, in der Glukose über Pyruvat zu Laktat umgewandelt wird. Dabei können pro Molekül Glukose nur 2 Moleküle ATP gebildet werden, im Gegensatz zu den 38 Molekülen ATP, die bei voller oxidativer Verstoffwechslung pro Glukosemolekül anfallen.

Der Gehalt an energiereichen Phosphaten im Herzen läßt sich in vivo mit der NMR-Spektroskopie bestimmen. Dabei wurden in gesunden Probanden für Kreatinphosphat $11 \pm 2,7 \mu \mathrm{mol} / \mathrm{g}$ Feuchtgewicht und für ATP $6,9 \pm 1,6 \mu \mathrm{mol} / \mathrm{g}$ Feuchtgewicht ermittelt; dies entspricht einem KP/ATP-Quotienten von ca. 2. Bei Patienten mit koronarer Herzkrankheit fand sich unter Ruhebedingungen ein im Vergleich zu Herzgesunden gering vermindertes myokardiales KP-ATP-Verhältnis von 1,45 \pm 0,31 . Unter Belastung sank diese Größe bei den Koronarkranken auf 0,91 $\pm 0,24$ ab, während sie bei den Herzgesunden konstant blieb. Bei Patienten mit Kardiomyopathie liefern die entsprechenden Untersuchungen uneinheitliche Ergebnisse; ein Abfall des KP/ATP-Quotienten scheint hier mehr von der Schwere der Herzinsuffizienz als von der zugrundeliegenden Form der Kardiomyopathie abzuhängen.

\subsection{3}

\section{Ischämiebedingte Zellfunktionsschädigung}

\section{Schock}

Die systemische und regionale Minderperfusion spielt als Verursacher der ischämisch bedingten Zellschädi- 
gung bei den meisten Schockformen eine prägende Rolle. Sind die neurohumoralen Adaptationsmechanismen (s. Abb. 33-13) nicht mehr in der Lage, die schockinduzierte Organischämie und Hypoxie zu kompensieren, so sind die Hemmung des aeroben Zellstoffwechsels, die Abhängigkeit der Energieproduktion von der allein nicht ausreichenden anaeroben Glykolyse, die daraus resultierende zelluläre Energieverarmung mit Laktatanstieg und Azidose die Folge.

Diese schwerwiegende ischämisch bedingte Zellschädigung ist jedoch keine simple Hypoxiefolge auf die mitochondriale ATP-Produktion allein: Die Mitochondrien zeigen auch noch bei sehr niedrigem $\mathrm{O}_{2}$-Partialdruck (Michaelis-Konstante $<1 \mu \mathrm{M}$ ) eine adäquate ATPProduktion. Demzufolge garantiert diese hohe $\mathrm{O}_{2}$-Affinität auch eine regelrechte Funktion der Atmungskette, selbst in der Hypoxie, sieht man von Extrembedingungen einmal ab. Neben der Hypoxie sind es noch andere Faktoren der Ischämie und neben der mitochondrialen Atmungskette weitere Komponenten der Substratutilisation und Energiegewinnung, die zu der ischämisch bedingten Zellschädigung im Schock führen (Übersicht in Werdan 1995a).

Der so gestörte Substrat- und Energiestoffwechsel verursacht zahlreiche Zellschäden, die sich als Destruktion von Mitochondrien, als Beeinträchtigung der strukturellen und funktionellen Zellmembranintegrität, in Form zytotoxischer Effekte und schließlich als Zelltod manifestieren (Haglund 1993). Leber und Niere scheinen auf einen Abfall des Zell-ATP besonders empfindlich zu reagieren.

\section{Sepsis und septischer Schock}

Zumindest in der Initialphase der Sepsis scheint eine ischämie- und hypoxiebedingte Zellschädigung nicht im Vordergrund zu stehen: Beim Einsatz von Hypoxiemarkern ( ${ }^{18} \mathrm{~F}$-Fluormisonidazol) ließ sich in einem Rattensepsismodell keine Gewebehypoxie erkennen, auch nicht im Skelettmuskel und im Herzen. Die meisten der durchgeführten Untersuchungen fanden keine erniedrigten ATP-Gehalte (ausgenommen in Leber und Niere in der späten Sepsisphase) trotz erhöhter arterieller Laktatspiegel.

Auch der Kreatinphosphatgehalt in Muskelbiopsien septischer Patienten ist nicht erniedrigt (Tresadern et al. 1988). Sollte tatsächlich der aerobe Stoffwechsel in der Sepsis eingeschränkt sein, so würde dies durch eine Steigerung der $\mathrm{O}_{2}$-Extraktionsrate, die im Skelettmuskel hoch und im Herzen weniger hoch ist, und durch eine Zunahme der anaeroben ATP-Produktion effektiv ausgeglichen werden. Erwartungsgemäß finden sich auch keine Hinweise für eine strukturelle Mitochondrienschädigung in der Sepsis.

Neben einem verminderten $O_{2}$-Verbrauch von Skelettmuskelmitochondrien von Patienten mit septischem Schock (Poderoso et al. 1978), einer Verminderung der Aktivität der Komplexe I und II der Atmungskette im septischen Herzen (Gellerich et al. 1999) und einer beschriebenen Hemmung der mitochondrialen Atmung durch Endotoxin wurde auch eine Steigerung des $\mathrm{O}_{2}$ Verbrauchs von Rattenlebermitochondrien bei Endotoxinschock gesehen.

In ähnlicher Weise widersprüchlich sind auch die Ergebnisse zur möglichen Beeinflussung des mitochondrialen Krebs-Zyklus in der Sepsis, der als eingeschränkt oder als unbeeinflußt beschrieben worden ist. Bei der Diskussion dieser Ergebnisse darf allerdings nicht vergessen werden, daß nicht nur Ischämie und Hypoxie zur Beeinträchtigung der mitochondrialen Funktion und ATP-Produktion führen können, sondern auch Mediatoren, insbesondere Zytokine (Tabelle 33-2; Pellicane et al. 1993; Zell et al. 1997).

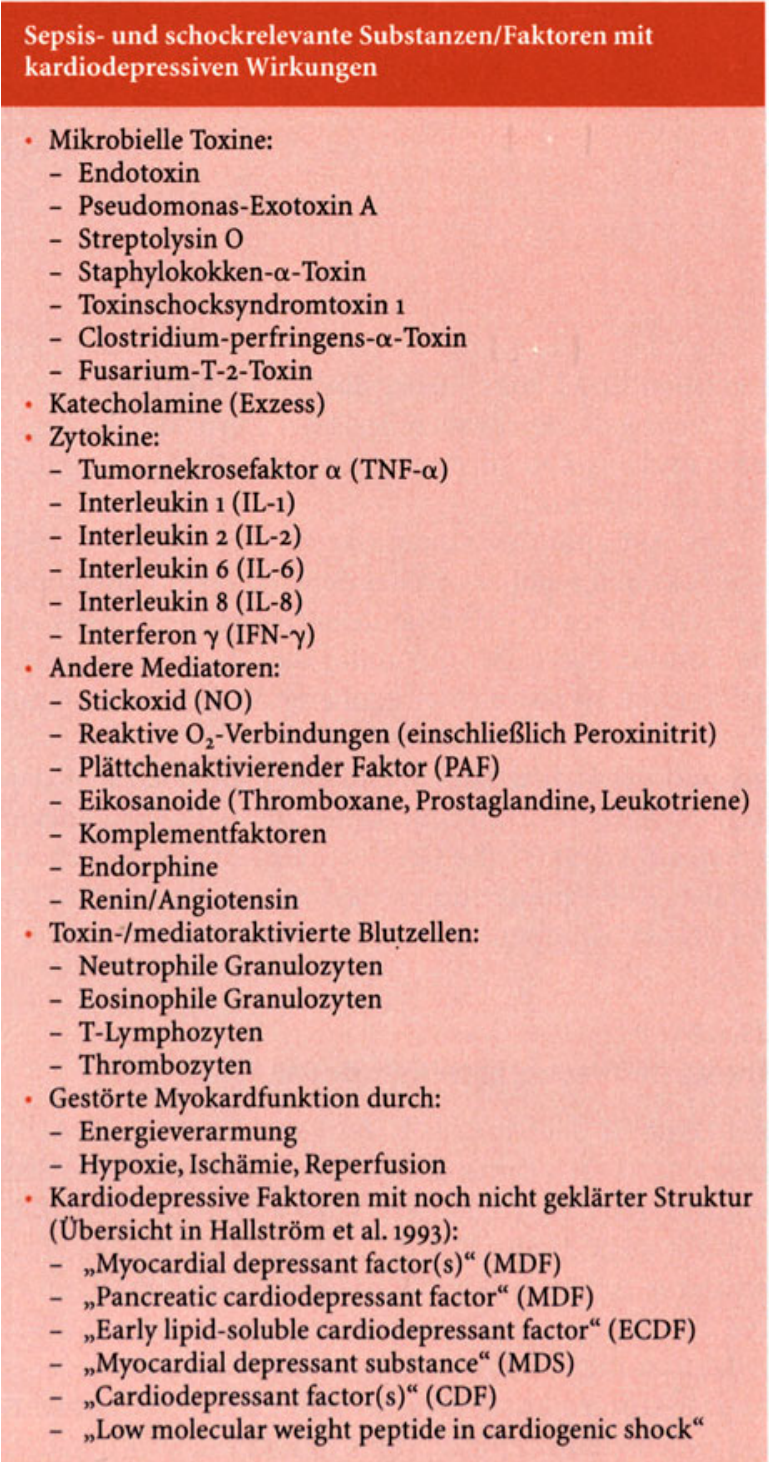

Der erhöhte Blutlaktatspiegel bei Patienten in Schock und Sepsis wird häufig ausschließlich als Ausdruck einer lokalen Hypoxie und Ischämie angesehen. Bei vergleich- 
Tabelle 33-2. Zytokine u.a. Mediatoren bei Herz-Kreislauf-Erkrankungen

\begin{tabular}{|c|c|c|}
\hline Herzerkrankung & Zytokine/Mediatoren & Wirkungen \\
\hline $\begin{array}{l}\text { Schock, } \\
\text { septische Kardiomyopathie }\end{array}$ & s. Abschn. 33.8 & \\
\hline \multirow[t]{4}{*}{ Myokarditis } & $\begin{array}{l}\text { IL-1 } \alpha,-\beta, \text { IL-6, TNF- } \\
\alpha\end{array}$ & Erhöhte Plasma-/Serumspiegel bei einigen Patienten \\
\hline & TNF- $\alpha$, IL-1, IL-6 & Pathogenetische Bedeutung bei der experimentellen Myokarditis \\
\hline & IFN- $\gamma$ & Protektiv bei Autoimmunmyokarditis \\
\hline & anti-TNF- $\alpha-\mathrm{AK}$ & Besserung der Autoimmunmyokarditis \\
\hline Dilatative Kardiomyopathie & iNOS $\uparrow / c N O S \downarrow \downarrow$ & Verminderte $\beta$-adrenerge Responsivität \\
\hline $\begin{array}{l}\text { Hypertrophische } \\
\text { Kardiomyopathie }\end{array}$ & G-CSF $\uparrow, T N F-\alpha \uparrow$ & Pathogenetische Bedeutung der erhöhten Plasma-/Serumspiegel? \\
\hline \multirow[t]{2}{*}{$\begin{array}{l}\text { Akuter Herzinfarkt, } \\
\text { instabile Angina }\end{array}$} & TNF- $\alpha$ & Plasma-/Serumspiegel $\uparrow$, bes. bei ausgedehnten Infarkten \\
\hline & sTNFRI, IL-1ra, IL-6 & Plasma-/Serumspiegel $\uparrow$ \\
\hline $\begin{array}{l}\text { Nach Operationen mit Herz- } \\
\text { Lungen-Maschine }\end{array}$ & TNF- $\alpha$, IL- 6, IL- 8 & $\begin{array}{l}\text { Erhöhte arterielle Plasmaspiegel nach hypothermem CPB können zur post- } \\
\text { operativen Myokardischämie und zu segmentalen Kontraktionsstörungen } \\
\text { beitragen }\end{array}$ \\
\hline $\begin{array}{l}\text { Ischämiebedingte } \\
\text { Arrhythmien }\end{array}$ & Endotoxin & Präkonditionierung reduziert Arrhythmien, evtl. über Induktion der iNOS \\
\hline \multirow{2}{*}{$\begin{array}{l}\text { Myokardischämie, } \\
\text { Ischämiereperfusions- } \\
\text { schädigung }\end{array}$} & $\begin{array}{l}\text { Adhäsionsmoleküle } \\
\text { TNF- } \alpha\end{array}$ & $\begin{array}{l}\text { Expression triggert Leukozyten-Endothel-Interaktion } \\
\text { Zumindest teilweise für Reperfusionsschädigung verantwortlich }\end{array}$ \\
\hline & NO & Reduzierte Freisetzung oder Aktivität bei Koronararterien \\
\hline \multirow[t]{3}{*}{ Schwere Herzinsuffizienz } & TNF- $\alpha$, IL-6 & Serumspiegel $\uparrow$ bei einigen Patienten \\
\hline & TNF- $\alpha$ & Erhöhte Serumspiegel korrelieren mit kardialer Kachexie \\
\hline & NO & $\begin{array}{l}\text { Gefäßfreisetzung von NO: basale Freisetzung: erhalten oder sogar verstärkt; } \\
\text { ACh-stimuliert: vermindert }\end{array}$ \\
\hline Essentielle Hypertonie & $\begin{array}{l}\text { G-CSF, GM-CSF } \\
\text { ROS } \\
\text { NO }\end{array}$ & $\begin{array}{l}\text { Plasmaspiegel gering erhöht bei wenigen Patienten? } \\
\text { Gesteigerte Produktion in zirkulierenden neutrophilen Granulozyten } \\
\text { Verminderte Produktion in zirkulierenden neutrophilen Granulozyten }\end{array}$ \\
\hline Transplantatabstoßung & $\begin{array}{l}\text { TNF- } \alpha \\
\text { IL-2 }\end{array}$ & $\begin{array}{l}\text { Plasmaspiegel } \uparrow \text {, Expression von TNF- } \alpha \text {-positiven Entzündungszellen } \\
\text { Plasmaspiegel } \uparrow \text {; Expression von CD4-IL-2-positiven Zellen }\end{array}$ \\
\hline \multirow[t]{2}{*}{ Kawasaki-Syndrom } & TNF- $\alpha$ & $\begin{array}{l}\text { Serumspiegel in akuter und subakuter Phase signifikant höher als in Abhei- } \\
\text { lung }\end{array}$ \\
\hline & TNF- $\alpha$ IL-1, IFN- $\gamma$ & $\begin{array}{l}\text { Mögliche Rolle bei der Entstehung der Gefäßschädigung; Induktion zirku- } \\
\text { lierender, zytotoxischer Antikörper gegen Endothelzellantigene }\end{array}$ \\
\hline
\end{tabular}

IL Interleukin: $T N F-\alpha$ Tumornekrosefaktor: $I F N-\gamma$ Interferon $\gamma$ anti-TNF- $\alpha$ - $4 K$ Antikörper: $i N O S$ induzierbare Stickoxidsvnthase: cNOS konstitutive Stickoxidsynthase; G-CSF Granulozytenkoloniestimulierender Faktor; GM-CSF Granulozyten- Makrophagenkoloniestimulierender Faktor: sT TFRI löslicher T:VFRezeptor I; $/ L$-lra Interleukin-1-Rezeptorantagonist: NO Stickoxid: ROS reaktive $\mathrm{O}_{2}-$ Verbindungen: ( $P B$ kardiopulmonaler By pass: $A\left(h^{\prime} h\right.$ Acetyleholin: $\uparrow$ erhöht: $\downarrow$ vermindert. Weitere Erläuterungen und Literaturhinweise s. Müller-Werdan et al. 1996: Parker 1995: Müller-Werdan u. Werdan 1999

barer Einschränkung des $\mathrm{O}_{2}$-Metabolismus $\left(\mathrm{O}_{2}\right.$-Transport, $\mathrm{O}_{2}$-Verbrauch, Skelettmuskel- $\mathrm{pO}_{2}$ ) finden sich im Tierexperiment jedoch bei septischem Schock höhere arterielle Laktatspiegel als bei kardial-obstruktivem Schock. Dies läßt vermuten, daß die Laktatproduktion in der Sepsis nicht nur Folge der eingeschränkten $\mathrm{O}_{2}$ Versorgung ist, sondern auch andere Ursachen haben kann. Allerdings sprechen nicht alle Befunde gegen eine Störung des Energiestoffwechsels in Schock und Sepsis. In der Frühphase der Sepsis blieben die ATP-Werte konstant und der intrazelluläre $\mathrm{pH}$-Wert ändert sich nicht wesentlich. Diese Ergebnisse weisen auf einen in der Sepsis erhöhten ATP-Umsatz zur Aufrechterhaltung des Ionengleichgewichts und/oder gesteigerte metabolische Prozesse mit den Kreatinphosphatspeichern als Energiepuffer hin. In Übereinstimmung damit konnten im Plasma von Sepsispatienten erhöhte Spiegel von ATP-Abbauprodukten nachgewiesen werden.

Weitere Argumente für eine globale Ischämie, oder zumindest für fleckförmige Ischämiegewebezonen sind die pathologische $\mathrm{O}_{2}$-Abhängigkeit in der Sepsis (s. Abschn. 33.9) und die Ausschwemmung von organischen Säuren 
aus ischämischem Gewebe bei mit Vasodilatatoren behandelten Patienten mit Sepsis und Multiorganversagen (Bihari et al. 1987). Bei kritischer Wertung aller Ergebnisse spricht einiges für das Vorliegen einer globalen oder zumindest regionalen Ischämie in der Sepsis, ohne daß dem jedoch eine dominierende Bedeutung in der Entstehung des septischen Schocks zuzukommen scheint.

\section{5}

Toxine

\subsection{1}

\section{Endotoxin}

Von den zahlreichen Toxinen menschenpathogener Bakterien kommen in der Sepsis und im septischen Schock dem Endotoxin gramnegativer Keime sowie den Superantigenen und den Porenbildnern grampositiver Erreger die größte Bedeutung zu. Endotoxin ist ein in der äußeren Zellmembran gramnegativer Bakterien lokalisiertes Lipopolysaccharid (LPS). Es besteht aus einer in der Zellmembran fest verankerten, konstanten Lipidkomponente, dem Lipid A, und einer Kohlenhydratkette mit einem äußeren und inneren Kernoligosaccharid (in der Zellmembran sitzend) und der variablen O-spezifischen Zuckerkette, die wie ein Haar aus der Oberfläche der Zellmembran hervorragt (Loppnow et al. 1993).

Endotoxin gelangt entweder durch Zerfall von Bakterien im Intravasalraum (auch nach Gabe von Antibiotika in unterschiedlichem Ausmaß) oder durch Translokation von Bakterien und Endotoxin aus dem primären Infektionsherd oder aus dem Darm in die Blutbahn. Es kann quantitativ im peripheren Blut nachgewiesen werden, sobald die Clearancekapazität des retikuloendotheli- alen Systems für Endotoxin erschöpft ist („,spillover“); es finden sich bei Patienten mit gramnegativem septischem Schock Endotoxinplasmaspiegel in der Größenordnung von 10-200 pg/ml. Dennoch ist der quantitative Nachweis von Endotoxin derzeit noch mehr von wissenschaftlichem als von praktisch-klinischem Interesse.

Zum Zeitpunkt einer systemischen Endotoxinämie haben die Endotoxine verschiedene Reaktionen in Gang gesetzt (Meßmer et al.1986), nämlich die pyrogene Reaktion, die Stimulation der humoralen Immunantwort, die Aktivierung des Gerinnungs-, Komplement- und Kallikrein-Kinin-Systems sowie verschiedene Freisetzungsreaktionen im Gewebe (biogene Amine, Proteasen und Peptide, Eikosanoide), z.T. vermittelt durch die Zytokine, Tumornekrosefaktor $\alpha$ (TNF- $\alpha)$ und die Interleukine 1 , 6 und 8 (IL-1, -6, -8; s. auch Abb. 33-5).

Diese Wirkungen entfaltet das Endotoxinmolekül durch eine spezifische, rezeptorvermittelte Bindung an Zielzellen (Schumann u. Rietschel 1995) mit Auslösung einer zellspezifischen Proteinexpression: Dazu lagert sich das zirkulierende Endotoxinmolekül zunächst an ein ebenfalls zirkulierendes Lipoproteinbindungsproteinmolekül (LBP) an (Abb. 33-6); in Monozyten-Makrophagen koppelt sich dieser LPS-LBP-Komplex an den Endotoxinrezeptor, der aus dem CD14-Molekül und einer weiteren Rezeptorkomponente besteht. Fehlt der Zielzelle ein membranständiger CD14-Rezeptor, muß sich der LPS-LBP-Komplex zunächst mit einem ebenfalls zirkulierenden, löslichen CD14-Molekül zusammenlagern, ehe sich dann dieser Komplex an die Zielzelle (z.B. eine Endothelzelle) anlagern kann. Die Folge der Endotoxinrezeptorbindung ist im Falle der Monozyten/ Makrophagen eine Zytokininduktion und -freisetzung; bei den Endothelzellen führt dies zur Expression von

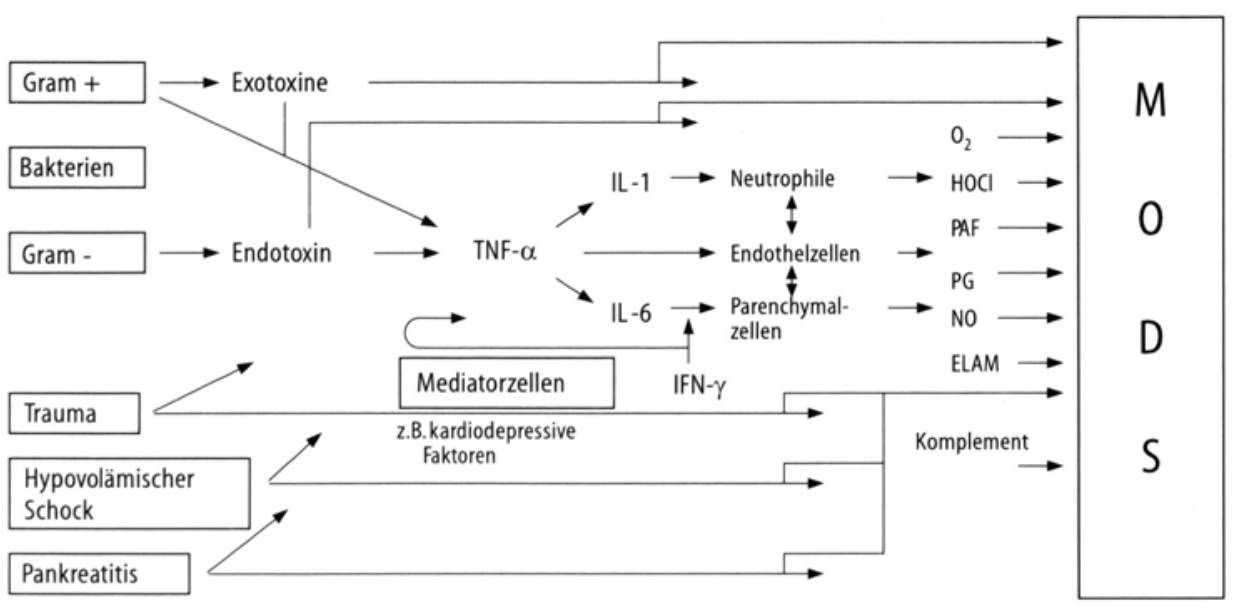

Ausloser

(Trigger)

Abb. 33-5. Toxin- und Mediatornetzwerke bei Sepsis und SIRS. INH-r Tumornekrosetaktor a; II,-1 Interleukin I; II,-6 Interleukin o; II: $\mathrm{N}$ - Interteron $\gamma ;$ Neutrophile neutrophile (iranulozyten; () superoxidanion; H()(./ hypochlorige Säure; PAl plätchenaktivie-
"Finale Mediatoren"

render Faktor; P(; Prostaglandine; NO Stickoxid; ELAM Adhäsionsmodell I:L.AM; MOI)S Multiorgandysfunktionssyndrom. (Mod. nach Müller-Werdan et al. 1996) 
Abb. 33-6. Mechanismus der Endotoxin-Rezeptor-Bindung in Makrophagen und Endothelzellen. LPS Lipopolysaccharid; $L B P$ Lipoproteinbindungsprotein; $C D_{14}$ membrangebundenes CD14-Rezeptormolekül; $s C D_{14}$ lösliches CD14-Rezeptormolekül; $I L-1$ Interleukin $1 ; I L-6$ Interleukin 6; TNF Tumornekrosefaktor. (Nach Schumann u. Rietschel 1995)
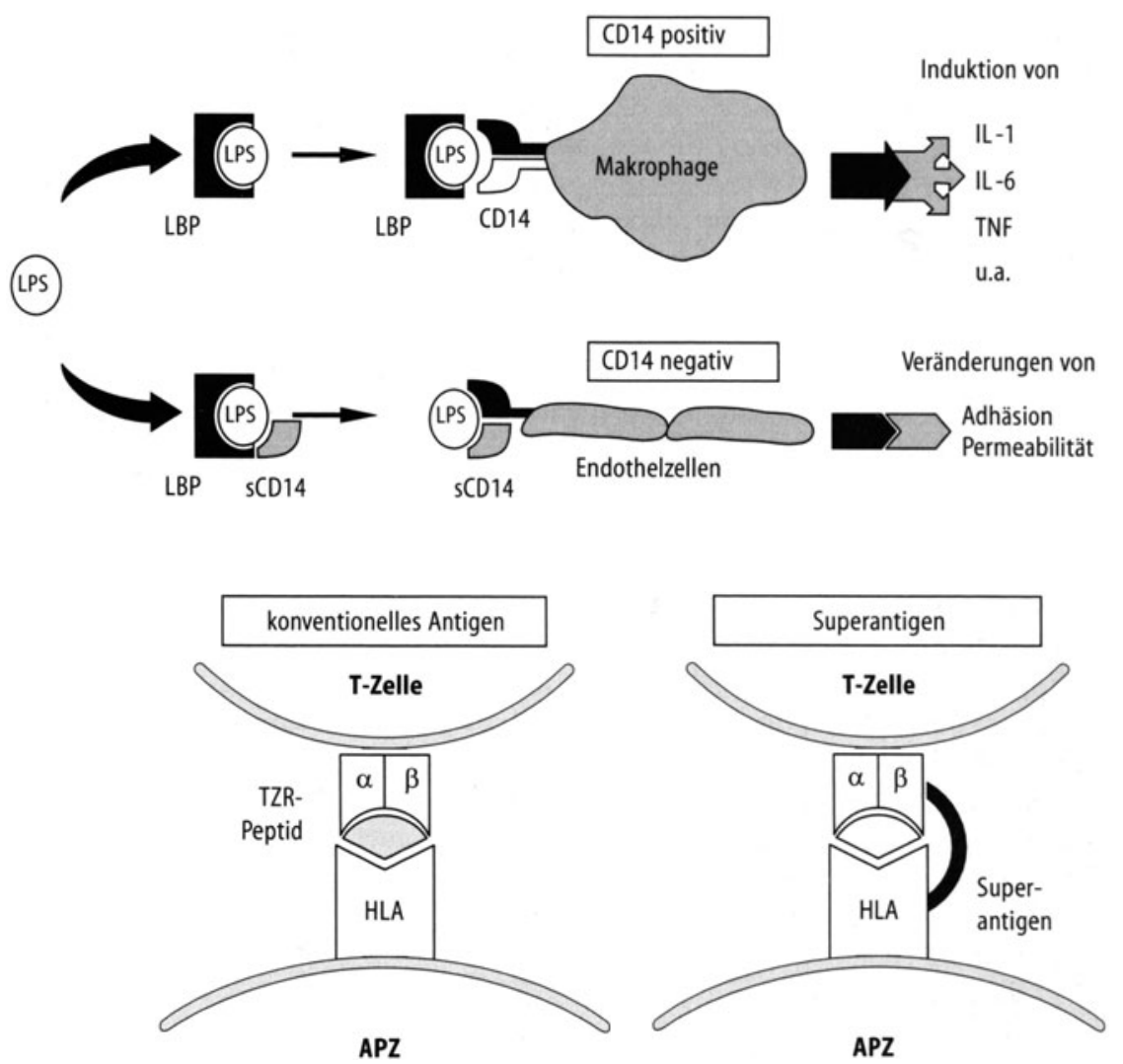

APZ
Abb. 33-7. Antigen- und Superantigenerkennung. Vereinfachte Darstellung der Antigenerkennung von $\mathrm{T}$-Zellen. TZR T-Zellrezeptor; $A P Z$ antigenpräsentierende Zelle; $H L A$ HLA-Klasse-II-Molekül; $a, \beta a$ bzw. $\beta$-Kette des T-Zellrezeptors. (Aus Heeg et al. 1995)
Adhäsionsmolekülen, was wiederum Voraussetzung für die Anlagerung von Blutzellen an das Endothel ist.

Endotoxin ist zweifellos ganz entscheidend für die schlechte Prognose bei gramnegativem Schock mitverantwortlich. Darüber sollte jedoch nicht vergessen werden, daß es in niedrigen Konzentrationen über eine nur moderate Mediatorfreisetzung bei der Infektabwehr durchaus günstig wirken kann (mäßiges Fieber, Steigerung der Immunabwehr, Abtötung der Keime). Die durch Endotoxin induzierte Hypotension scheint mit der Zeit eine Abschwächung zu erfahren (Mailman et al. 1999).

Aus kardiologischer Sicht bietet das Endotoxin auch Überraschungen: neben der deletären direkten und indirekten kardiodepressiven Wirkung (s. Abschn. 33.8) lassen sich protektive Effekte auf das Herz nachweisen (Song et al. 1994; Yao et al. 1993; McDonough et al. 1995).

\subsection{2}

\section{Superantigene}

Grampositive Erreger verursachen mindestens ebenso viele Sepsiserkrankungen wie gramnegative Keime (Bone 1994).

Da grampositive Bakterien keine Lipopolysaccharide synthetisieren und in ihre Zellwand integrieren, schei- det Endotoxin als Induktor einer grampositiven Sepsis aus. Hierfür kommen einerseits Peptidoglykane, die wie Endotoxin an CD14 binden können, und die Lipoteichonsäuren der Zellmembran in Frage und andererseits Toxine, die entweder als Superantigene oder als Porenbildner ihre zytotoxische Wirkung entfalten. Peptidoglykane und Lipoteichonsäuren wirken pyrogen, aktivieren Komplement und B-Lymphozyten. Dabei werden die Makrophagen aktiviert und sezernieren Zytokine, teils in größeren Mengen als nach Endotoxinstimulation.

Toxine mit Superantigeneigenschaften (Abb. 33-7), wie z.B. das Toxinschocksyndromtoxin 1 bestimmter Staphylokokken, besitzen wie konventionelle Antigene die Fähigkeit, T-Lymphozyten zu aktivieren. Unter Umgehung bestimmter Kontrollmechanismen stimulieren sie jedoch nicht nur $0,01 \%$ der Zellen, wie konventionelle Antigene, sondern 2-10-25\% aller T-Lymphozyten gleichzeitig.

Die Folge ist eine Überaktivierung der T-Zellen mit massiver Zytokinfreisetzung, insbesondere TNF- $\alpha$, und evtl. letalem Schockverlauf (Heeg et al. 1995).

Die Bedeutung der Superantigene bei der Pathogenese der Sepsis kann gegenwärtig noch nicht abgeschätzt werden. Beim Menschen könnte das vor einigen Jahren erstmals beschriebene toxische Schocksyndrom ein Prototyp der superantigenvermittelten Sepsis sein. Dabei 
kommt es zu einer lokalen Infektion mit Staphylococcusaureus-Stämmen, die das Toxinschocksyndromtoxin 1 (TSST-1) produzieren (s. Abschn. 37.2.2). Weitere Staphylokokkensuperantigene sind die Enterotoxine A-E und das exfoliative Toxin. Mehr als 40\% aller klinischen Staphylococcus-aureus-Isolate produzieren eines oder mehrere Superantigene.Andererseits können auch pathogene Staphylokokkenstämme isoliert werden, die keines der bekannten Superantigene produzieren. Ein weiteres Superantigen ist das erythrogene Toxin A der Streptokokken. Superantigene sind jedoch nicht auf die grampositiven Bakterien beschränkt: Pseudomonas (Exotoxin A), Yersinien, Mykobakterien und sogar das HI-Virus können Superantigene produzieren (Heeg et al. 1995).

Superantigene und Endotoxin können sich in ihrer Wirkung verstärken: Bei gleichzeitigem Vorhandensein beider Toxinklassen reicht die Kombination bereits sehr geringer Toxinmengen zur Schockauslösung bei Tieren aus. Da es auch bei grampositiver Sepsis und bei nichtinfektiösen Schockformen infolge einer bakteriellen Translokation im Darm zur Endotoxinämie kommen kann, kommt dieser Toxininteraktion möglicherweise auch eine klinische Bedeutung $\mathrm{zu}$.

\subsection{3}

\section{Porenbildner}

Porenbildner sind von Bakterien sezernierte Proteintoxine, die an Zellmembrankomponenten (vorwiegend Lipide) binden und auf diese Weise die Zellmembran mit Ausbildung 1-30 nm großer Löcher schädigen (Seeger et al. 1993; Bhakdi et al. 1989).

Prototypen dieser Toxinklassen sind das $\alpha$-Toxin von Staphylococcus aureus, das Streptolysin-O von Staphylococcus pyogenes A und das E.-coli-Hämolysin neben mindestens 14 anderen Toxinen grampositiver und mindestens 11 gramnegativer Bakterien. Ihre klinische Relevanz, v.a. bei grampositiven Infektionen, wird derzeit wahrscheinlich noch unterschätzt. Insbesondere die prokoagulatorische Wirkung auf das Gerinnungssystem und die Endothelzellschädigung mit der Ausbildung einer pulmonalen Hypertonie scheinen wesentliche Kompo- nenten der Schockauslösung durch die Porenbildner zu sein.

\section{6}

\section{Mediatoren}

Das Verständnis um die Bedeutung von Toxinen und Mediatoren im Schockgeschehen fußte zunächst auf Erkenntnissen, die bei der Aufklärung der Pathogenese des septischen Schocks gewonnen worden sind (Abb. 33-5). Heutzutage kann jedoch davon ausgegangen werden, daß die Mediatorkaskade die gemeinsame Endstrecke nicht nur des septischen Schocks darstellt (Abb. 33-8), sondern daß auch ein hypovolämischer, traumatischer, anaphylaktischer und sogar der kardiogene Schock Komponenten dieser Kaskade als auslösende oder unterhaltende Ursachen beinhalten.

Dieses Konzept spiegelt sich auch in der aktuellen Terminologie von Sepsis und SIRS (Systemic inflammatory response syndrome, Tabelle 32-3) wider: Ausgehend von infektiösen oder nichtinfektiösen Stimuli kommt es zur Aktivierung von Mediatorzellen, die primäre Mediatoren wie den Tumornekrosefaktor $\alpha$ freisetzen. Diese primären Mediatoren beeinflussen weitere Zielzellen, die einerseits geschädigt werden können, andererseits (wie z..B. die neutrophilen Granulozyten) finale Mediatoren auf diesen Stimulus (wie z.B. reaktive $\mathrm{O}_{2}$-Verbindungen oder Stickoxid) freisetzen. Die Mediatorfreisetzung soll zwar eigentlich zur Bekämpfung der Bakterien, zur Neutralisierung von Bakterientoxinen und zur Schadensbegrenzung nichtinfektiöser Insulte dienen, die aggressiven Verbindungen schädigen dabei jedoch auch das Herz-Kreislauf-System und weitere vitale Organe: eine Verschlimmerung der Schocksymptomatik und die Ausbildung eines Multiorgandysfunktionssyndroms sind die Folgen.

\subsection{1 \\ Zytokine}

Zytokine, insbesondere TNF- $\alpha$ und IL-1, stehen am Anfang des Mediatornetzwerks von Schock und Sepsis. Bak-
Abb. 33-8. Pathogenesekonzept der gramnegativen und grampositiven Sepsis. $I L,-1$, $-2,-4,-6$ Interleukin $1,2,4$, 6; T'/R T-Zellrezeptor; LPS Lipopolysaccharid; (II) (II)14Rezeptormolekül; TNF Tumornekrosefaktor. (Aus Heeg et al. 1995)

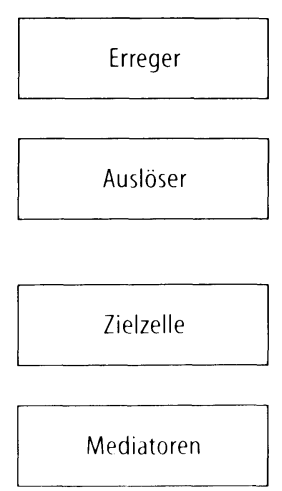


Tabelle 33-3. Stickoxid (NO) und Schock. eNOS endotheliale Stickoxidsynthase; iNOS induzierbare Stickoxidsynthase; TNF- $\alpha$ Tumornekrosefaktor $\alpha$; IL-1 Interleukin 1; IFN- $\gamma$ Interferon $\gamma$; PAF plättchenaktivierender Faktor. (Mod. nach Thiemermann 1995)

\begin{tabular}{|c|c|c|c|c|}
\hline Schockart & $\begin{array}{l}\text { Werte im } \\
\text { Patientenplasma }\end{array}$ & Initialphase & Nach Stunden & Prolongierter Schock \\
\hline Septischer Schock & $\begin{array}{l}\text { Nitrit } \uparrow \\
\text { Nitrat } \uparrow\end{array}$ & $\begin{array}{l}\text { eNOS- } \\
\text { Aktivierung } \\
\rightarrow \text { Hypotonie }\end{array}$ & $\begin{array}{l}\text { iNOS-Induktion durch TNF- } \alpha, \mathrm{IL}-1, \\
\text { IFN- } \gamma \text { und PAF } \\
\rightarrow \text { exzessive NO-Produktion durch } \\
\text { iNOS in Gefäßwand } \\
\rightarrow \text { Vasodilatation } \\
\rightarrow \text { Gefäßhyporeagibilität auf Vasokon- } \\
\text { striktoren (Noradrenalin u.a.) } \\
\rightarrow \mathrm{O}_{2} \text {-Diffusionsbarriere (verminderte } \\
\mathrm{O}_{2} \text {-Extraktion) durch } \mathrm{O}_{2} \text {-Verbrauch } \\
\quad \text { (Bildung von Nitrit und Nitrat aus } \\
\text { NO und } \mathrm{O}_{2} \text { ) } \\
\rightarrow \text { Kreislaufschock mit Minder- und- } \\
\text { Fehlperfusion sowie reduzierter } \mathrm{O}_{2} \text { - } \\
\text { Extraktion } \\
\rightarrow \text { exzessive NO-Produktion durch } \\
\text { iNOS im Myokard } \\
\rightarrow \text { Myokarddepression (s. Abschn. } 33.8 \text { ) } \\
\rightarrow \text { exzessive NO-Produktion durch } \\
\text { iNOS lokal (Organe) } \\
\rightarrow \text { verminderte } \mathrm{O}_{2} \text {-Extraktion durch } \\
\text { zelluläre } \mathrm{O}_{2} \text {-Verwertungsstörung } \\
\text { (Hemmung der mitochondrialen At- } \\
\text { mung durch NO) } \\
\rightarrow \text { Zytotoxizität durch Bildung von } \\
\text { Peroxinitritradikalen }\end{array}$ & $\begin{array}{l}\text { Prolongierte Endotheldys- } \\
\text { funktion } \\
\text { (Beeinträchtigung der endo- } \\
\text { thelabhängigen Vasodilata- } \\
\text { tion infolge verminderter } \\
\text { NO-Produktion) durch } \\
\rightarrow \text { Downregulation von eNOS } \\
\quad \text { durch TNF u.a. Zytokine } \\
\rightarrow \text { Endothelzellschädigung } \\
\text { infolge zytotoxischer NO- } \\
\text { Wirkungen } \\
\rightarrow \text { Inaktivierung von NO } \\
\quad \text { durch } \mathrm{O}_{2} \text { Radikale }\end{array}$ \\
\hline $\begin{array}{l}\text { Hämorrhagischer } \\
\text { Schock }\end{array}$ & $\begin{array}{l}\text { Nitrit } \uparrow \\
\text { Nitrat } \uparrow\end{array}$ & & $\begin{array}{l}\text { iNOS-Induktion in der Gefäßwand } \\
\rightarrow \text { exzessive NO-Produktion durch } \\
\text { iNOS } \\
\rightarrow \text { Gefäßhyporeagibilität gegenüber Va- } \\
\quad \text { Vasokonstriktoren (Noradrenalin u.a.) }\end{array}$ & s. septischer Schock \\
\hline $\begin{array}{l}\text { Anaphylaktischer, } \\
\text { traumatischer und } \\
\text { Verbrennungs- } \\
\text { schock }\end{array}$ & Nitrit $\uparrow$ & & $\begin{array}{l}\text { Pathogenetische Bedeutung von NO } \\
\text { unklar }\end{array}$ & \\
\hline
\end{tabular}

terielle Toxine, aber auch SIRS-Stimuli, können Monozyten und Makrophagen zur Bildung und Freisetzung insbesondere von TNF- $\alpha$ aktivieren (Abb. 33-5).

Als Resultat der direkten und indirekten Zytokinwirkungen stehen beim septischen Schock die häufig irreversible Herz-Kreislauf-Schädigung und das Multiorgandysfunktionssyndrom. Die komplexen Wirkungen der Zytokine sind ausschnittsweise in Tabelle 33-3 wiedergegeben, ihre bekannte Bedeutung auf den Zellstoffwechsel in der Sepsis ist in Abschn. 33.7 beschrieben, und zur Kardiodepression führende Zytokineffekte sind in Abschn. 33.8 zusammengefaßt.

Die Bedeutung der Zytokine beschränkt sich jedoch nicht nur auf den septischen Schock: auch beim hämorrhagischen Schock finden sich erhöhte TNF-Serumspiegel, und das akute Lungenversagen bei hypovolämischem und traumatischem Schock läßt sich durch den Einsatz von Anti-TNF-( $x$-Antikörpern bessern. Erhöhte Zytokinserum-/Plasmaspiegel finden sich darüber hinaus auch bei zahlreichen infektiösen und nichtinfektiösen Herz-Kreislauf-Erkrankungen. Derzeit muß al- lerdings noch häufig die Frage offenbleiben, ob Zytokine dabei kausale Bedeutung haben, ein sekundäres Phänomen von Krankheitswert oder nur ein unwichtiges Epiphänomen darstellen (ausführliche Diskussion in Müller-Werdan et al. 1996).

Das Verstehen der Zytokinwirkmechanismen (Williams u. Giroir 1995; Ibelgaufts 1995) - gezeigt am Beispiel des TNF- $\alpha$ - bietet die Möglichkeit einer kausalen Therapie, z.B. mit Anti-TNF- $\alpha$-Antikörpern. An der Zielzelle bindet TNF- $\alpha$ (als Trimer) an einen der beiden TNF-Rezeptoren (TNFR1, MG 55000; TNFR2, MG 75000) und löst damit verschiedene zelluläre Signale aus (Abschn. 33.8). Unterschiedliche Stimuli (Fieber, Endotoxin, TNF- $\alpha$ selbst und andere Zytokine) können zur proteolytischen Spaltung der TNF-Rezeptoren und ihrer Freisetzung als lösliche TNF-Rezeptoren ins Plasma führen. Deren Bedeutung ist jedoch bisher noch unklar: In niedrigen Konzentrationen scheinen sie ein Reservoir für das gebundene TNF-c darzustellen, in höheren Konzentrationen das TNF- $x$ und damit seine zytotoxische Wirkung zu neutralisieren. 
Zytokine sind nicht nur schädlich; es ist vielmehr die massiv gesteigerte Produktion und damit die Dysbalance von proinflammatorischen Mediatoren wie TNF, IL-1, IL-2, IL-8, IFN und dem plättchenaktivierenden Faktor mit den antiinflammatorischen Substanzen und Mechanismen (IL-10, Interleukin-1-Rezeptorantagonist, lösliche TNF-Rezeptoren, Glukokortikoide, und auch Fieber), die letztlich bei Schock und Sepsis die ungünstige Prognose determiniert. So zeigt eine hohe Interleukin6/Interleukin-10-Relation bei kritisch Kranken eine ungünstige Prognose an (Taniguchi et al. 1999). Die Tatsache, daß niedrige Zytokin-, z.B. TNF- $\alpha$, Konzentrationen durchaus bei Sepsis zur Abwehrreaktion in protektiver Weise beitragen können, belastet das kausale Therapiekonzept der Unterbrechung dieser Zytokin-/ Mediatorkaskade.

\section{Zytokin-Genpolymorphismen}

Die Prognose eines Schockpatienten hängt wahrscheinlich nicht nur von seinem Phänotyp, sondern auch von seinem Genotyp ab, wie bisher v.a. für Patienten mit Sepsis und septischem Schock gezeigt worden ist (Stokkers et al. 1996):

Sepsispatienten, die homozygot sind für das Allel TNFB2 des TNF-Polymorphismus (NcoI-Restriktionsfragmentlängenpolymorphismus), produzieren höhere TNF-Plasmaspiegel und haben eine ungünstigere Prognose als die Sepsispatienten mit dem Genotyp TNFB1/B1

Abb.33-9. Entstehung von $\mathrm{O}_{2}-$ Radikalen und reaktiven Verbindungen. * Enzymbesatz neutrophiler Granulozyten; $\mathrm{O}_{2}^{-*}$ Superoxidanionradikal; $\mathrm{OH}^{\circ}$ Hydroxylradikal; $\mathrm{H}_{2} \mathrm{O}_{2}$ Wasserstoffperoxid; GSSG oxidiertes Glutathion; NO Stickoxid; $\mathrm{ONOO}^{-}$Peroxinitrit. (Mod. nach Wagenknecht et al. 1989) bzw. TNFB1/B2 (Stüber et al. 1996). Verknüpft mit dem TNF-Polymorphismus und ohne eigenständige prognostische Aussagekraft sind Polymorphismen der Hitzeschockproteine HSP7o-HOM C/T und HSP7o-2 G/A bei Patienten mit schwerer Sepsis (Schroeder et al. 1999).

Während sich für die Genotypverteilung des Interleukin $1 \beta$ keine Unterschiede zwischen Sepsispatienten und Gesunden zeigen, findet sich das Allel A2 des Interleukin 1-Rezeptorantagonisten (IL-1ra) bei Patienten mit schwerer Sepsis häufiger als bei Gesunden (Fang et al. 1999). Dies könnte auf eine erhöhte Sepsisempfindlichkeit bei dieser Allelkonstellation hinweisen.

\subsection{2}

\section{Reaktive Sauerstoffverbindungen (ROS)}

Reaktive $\mathrm{O}_{2}$-Verbindungen stellen im Schockgeschehen eine wichtige Gruppe terminaler Mediatoren dar.

Dabei handelt es sich um Moleküle mit einem ungepaarten Elektron in der äußeren Hülle. Ursprungsort von freien Radikalen sind zum einen aktivierte Mediatorzellen wie neutrophile Granulozyten, Makrophagen und Endothelzellen sowie andererseits (in der Reperfusionsphase der Schockbehandlung) das Hypoxanthin als Abbauprodukt des ATP. Die einzelnen Stoffwechselschritte gehen aus Abb. 33-9 hervor.

Peroxinitrit ist ein potentes und hochreaktives Oxidans mit ausgeprägter zytotoxischer Wirkung, das beson-
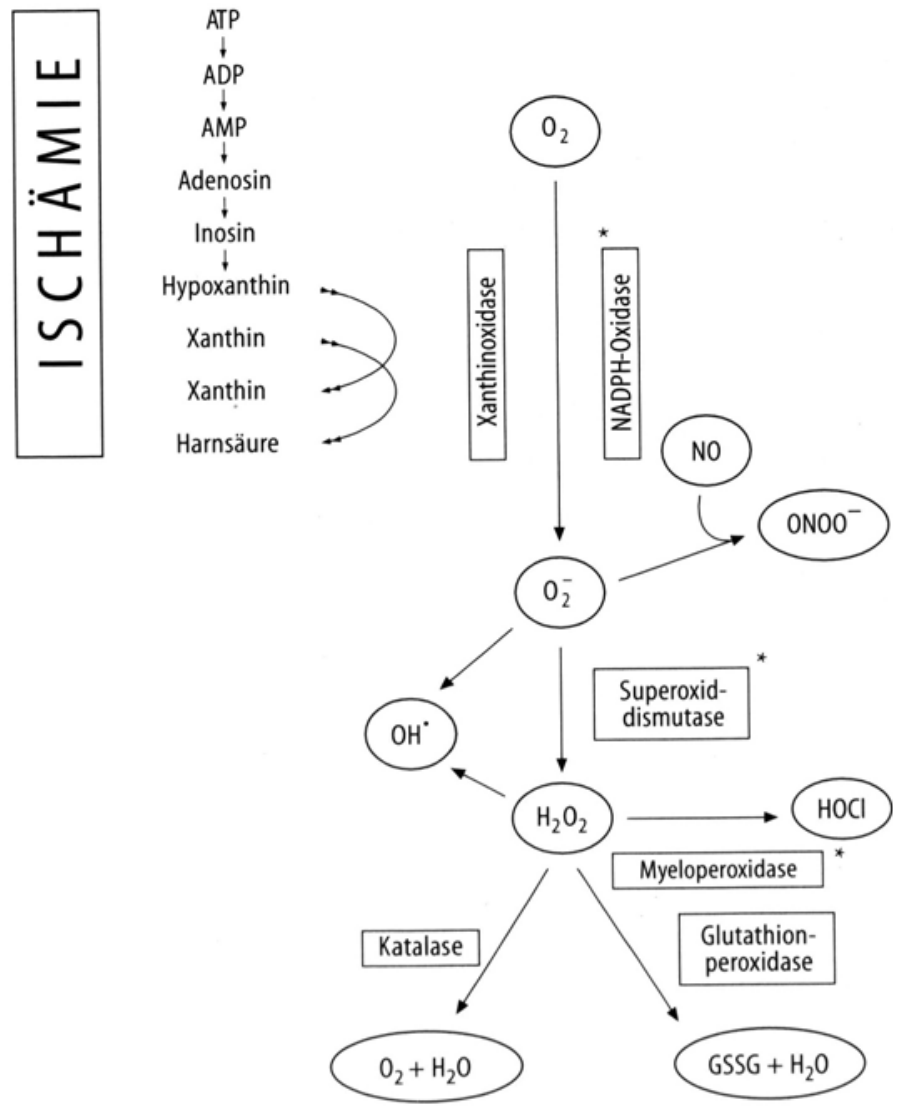


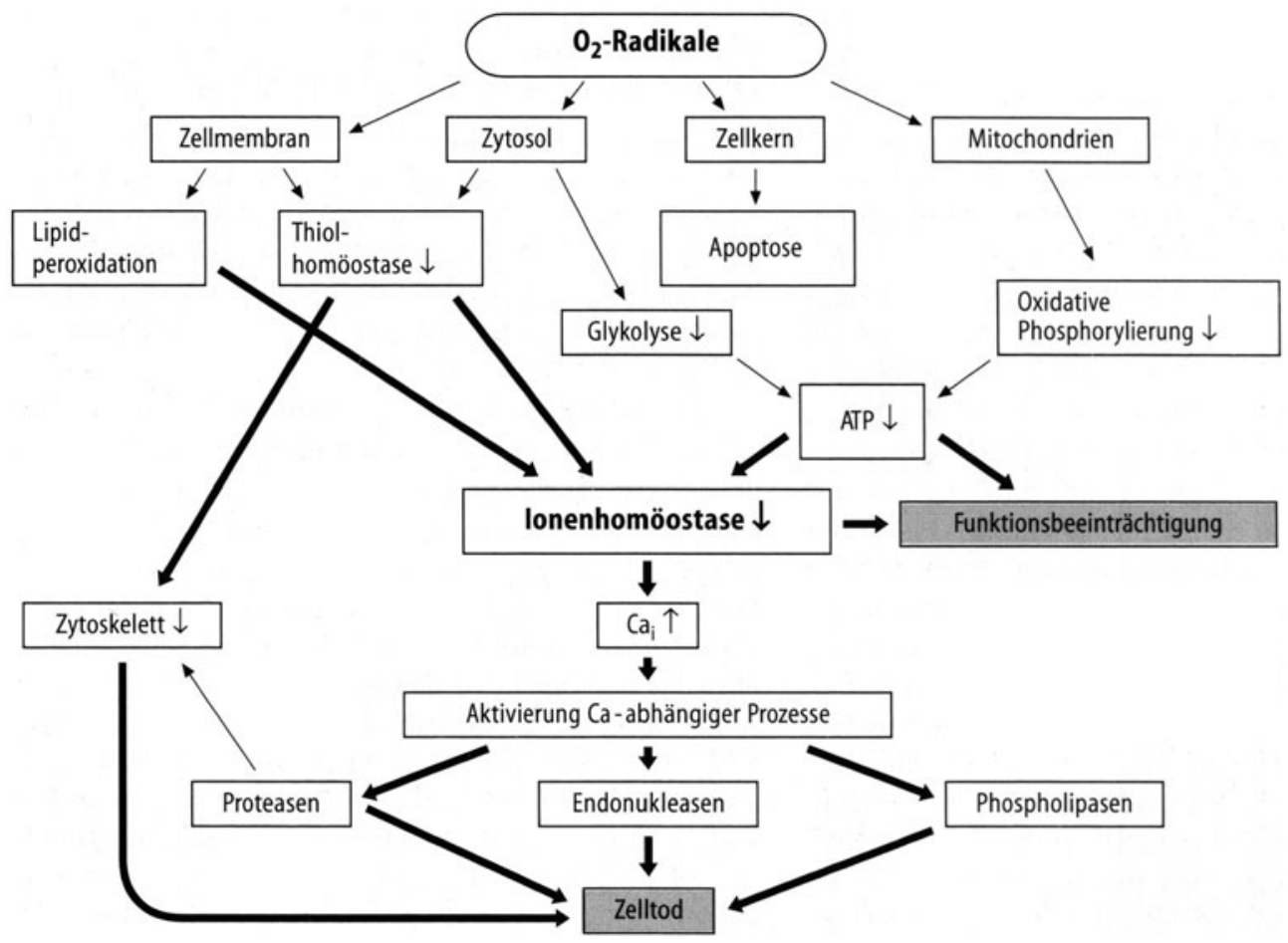

Abb. 33-10. Zelluläre Wirkungen von $\mathrm{O}_{2}$-Radikalen. (Mod. nach Wagenknecht et al. 1989)

ders zur Endothelschädigung führen kann, die Thrombozytenaggregation steigert und die Ansprechbarkeit der Koronargefäße auf Vasodilatatoren vermindert (Villa et al. 1994).

Treten die so entstandenen $\mathrm{O}_{2}$-Radikale bzw. reaktiven Verbindungen in Kontakt mit einer Zielzelle, so können sie auf verschiedene zelluläre Strukturen wirken: auf die Zellmembran, das Zytosol, den Zellkern und die Mitochondrien (Abb. 33-10). Schließlich wird durch diesen oxidativen Streß eine Reihe von Genen aktiviert („stress-response genes“), die zur Bildung von Antioxidanzien, $\mathrm{O}_{2}$-radikalabbauenden Enzymen, Zytokinen und Transkriptionsfaktoren führen. Sie dienen einerseits der Reparatur von Zellschäden durch die $\mathrm{O}_{2}$-Radikale und führen, falls dies nicht möglich ist, zum gerichteten Zelltod, der Apoptose (Camhi et al. 1995).

\subsection{3}

\section{Stickstoffmonoxid (NO)}

Das zunächst als „endothelial-derived relaxing factor“ klassifizierte Stickoxid (NO) wird enzymatisch aus Arginin gebildet. 3 Isoenzymformen der NO-Synthase sind dazu in der Lage: 2 konstitutive Formen (cNOS), die zur Aktivierung die Bindung von Calmodulin an das Enzym nach einer Anhebung des Zell-Ca ${ }^{2+}$ benötigen, und eine induzierbare, $\mathrm{Ca}^{2+}$-unabhängige Form, die permanent Calmodulin gebunden hat (iNOS).
Die cNOS-Isoformen sind ständig vorhanden. Sie können sofort aktiviert werden und produzieren geringe Mengen an NO, welches dann zahlreiche physiologische Funktionen erfüllt. Die cNOS-Isoenzyme können weiterhin in die neuronale Form (nNOS, lokalisiert im Zytosol von zentralen und peripheren Neuronen) und in die endotheliale Form (eNOS, überwiegend membrangebunden) eingeteilt werden. Die eNOS-Isoform besitzt sowohl parakrine als auch autokrine Aktivität; sie wird überwiegenß durch den Scherstreß der Gefäße aktiviert, das gebildete NO diffundiert in die glatten Gefäßmuskelzellen und führt zur Erschlaffung. Die eNOS ist nötig, um die Gefäße in einem partiell relaxierten Zustand zu halten, der durch Noradrenalin und Endothelin antagonisiert wird. Sie reguliert damit den Gefäßtonus, die Organperfusion und den Blutdruck. Weiterhin hemmt das von der eNOS produzierte NO die Adhäsion von Thrombozyten und Neutrophilen an Endothelzellen und möglicherweise auch die Proliferation von Gefäßmuskelzellen.

Die induzierbare Form der Stickoxidsynthase wurde zuerst in Makrophagen gefunden, sie kommt jedoch in vielen Zelltypen vor. Ihre Induktion durch zahlreiche Triggersubstanzen (s. Tabelle 33-3) dauert einige Stunden, das Enzymmolekül ist relativ langlebig (Halbwertszeit mehrere Stunden), und es kann durch die iNOS-Aktivität in kurzer Zeit lokal sehr viel Stickoxid gebildet werden. Demzufolge kann das zur Verfügung stehende Enzymsubstrat Arginin die Produktion von Stickoxid 
durch iNOS limitieren, was im Falle der wesentlich geringeren NO-Produktionsraten durch die cNOS-Aktivität nicht der Fall ist.

Ein dritter, für das Schockgeschehen möglicherweise sehr bedeutungsvoller Bildungsweg ist die nichtenzymatische Reduktion von Nitrit zu NO in minderdurchblutetem Gewebe: Im ischämischen, azidotischen Myokard findet sich Nitrit in mikromolaren Konzentrationen. Mittels Elektronenspinresonanzspektren ließ sich nachweisen, daß in diesem hochreduktiven Stoffwechselmilieu Nitrit in großen Mengen nichtenzymatisch zu Stickoxid reduziert wird (Zweier et al. 1995).

Das entstandene Stickoxid kann entweder am Ort der Bildung reagieren (autokrine Wirkung) oder in benachbarte Zellen diffundieren (parakrine Wirkung). Ein Großteil der Stickoxidwirkungen (Vasodilatation, Kardiodepression) beruht auf Aktivierung der löslichen Guanylatcyclase durch Stickoxid mit Bildung des zyklischen Guanosinmonophosphats (cGMP), des Gegenspielers des zyklischen Adenosinmonophosphats (cAMP). Bildung und Wirkung des Stickoxids können auf verschiedenen Stufen blockiert werden: Die Induktion der iNOS kann durch Glukokortikoide unterdrückt werden; die Stickoxidsynthasen lassen sich durch Argininanaloga wie L-NMMA ( $\mathrm{N}^{\mathrm{G}}$-Monomethyl-L-Arginin) hemmen, und die Aktivität der Guanylatcyclase kann durch Methylenblau blockiert werden.

In der Schockauslösung und der Schocktherapie ist Stickoxid ein entscheidender Mediator (Tabelle 33-3; Szabo u. Thiemermann 1994; Lorente et al. 1993; Parrat u. Stoclet 1995; Shears u. Bilia 1995). Die Hemmung der zellulären $\mathrm{O}_{2}$-Verwertungsstörung in den Organen durch NO-Inhibition einer Reihe mitochondrialer Enzyme (Aconitase, NADH-Ubichinon-Reduktase, SuccinatUbichinon-Oxidoreduktase) könnte dabei eine Ursache für die erhöht gefundenen $\mathrm{O}_{2}$-Partialdrücke im Skelettmuskel von Sepsispatienten (Tabelle 32-4) sein (Szabo u. Thiemermann 1994).

\subsection{4}

\section{Weitere Mediatoren}

Wie Abb. 33-5 zeigt, sind in der Schockpathogenese noch zahlreiche weitere Mediatoren zusätzlich zu den bisher beschriebenen involviert. Der Leser sei diesbezüglich auf weiterführende Literatur verwiesen (Schlag u. Redl 1993; Müller-Werdan et al. 1996; Pannen u. Robotham 1995; Müller-Werdan u. Schuster 2000). Neuere Aspekte betreffen die Apoptose (den gerichteten Zelltod) und das Spektrum der Hitzeschockproteine im Schockgeschehen sowie die Bedeutung der Adhäsionsmoleküle bei der Interaktion von Leukozyten mit der Mikrozirkulation bzw. mit Kardiomyozyten. Obwohl derzeit noch ohne praktische Relevanz, sind in Zukunft von der Aufklärung dieser Anpassungs- und Schädigungsmechanismen in der Frühphase des Schocks therapeutische Fort schritte zu erwarten.

\section{7}

Zellstoffwechsel und Apoptose im septischen Schock

Die komplexen Störungen des Zellstoffwechsels im septischen Schock sind nur teilweise Folge von Hypoxie und Ischämie; Toxine, Zytokine und andere Mediatoren und die neurohumorale Adaptations- und Dysregulation sind daran ebenfalls entscheidend beteiligt (Übersicht und Literatur in Müller-Werdan et al. 1996; Schmidt et al. 1999).

Eiweiß-, Kohlenhydrat- und Fettstoffwechsel sind im septischen Schock in charakteristischer Weise verändert: für die gesteigerte Proteolyse wird das Interleukin 1 verantwortlich gemacht, zusammen mit der Wirkung erhöhter Glukagon- und Cortisolspiegel. Dieser gesteigerte Proteinkatabolismus betrifft zwar vorwiegend den Skelettmuskel (und damit auch die Atemmuskulatur), er dürfte jedoch auch für den Herzmuskel nicht ohne Folgen sein. Die anfallenden Aminosäuren werden einerseits zur Synthese von Akutphaseproteinen in der Leber und andererseits für die Glukoneogenese genutzt. Die erhöhten Glukosespiegel könnten in ausreichender Menge das Substrat für glukoseabhängige Zellen zur Verfügung stellen.

Allerdings wird die Glukoseaufnahme des wesentlichen glukoseverbrauchenden Körperkompartiments, des Skelettmuskels, in der Sepsis zunehmend gehemmt. Ein wesentlicher Grund dafür ist die verminderte Aktivität des Pyruvatdehydrogenasekomplexes (PDH) des Skelettmuskels bereits in der frühen Sepsisphase, während die Aktivität der Leber-PDH erst bei höheren Sepsisschweregraden gehemmt wird. Die Hemmung der Pyruvatoxidation im Skelettmuskel führt zur Freisetzung von Pyruvat und Laktat, das in der Leber zur Glukoneogenese verwendet wird. Die gesteigerte Lipolyse ist im septischen Schock überwiegend die Folge der erhöhten Katecholamin-, Cortisol- und Glukagonspiegel. Der Tumornekrosefaktor hemmt die endotheliale Lipoproteinlipase und trägt dadurch zur Erhöhung der Triglyzeridspiegel bei.

Ein Beispiel für die dramatischen Stoffwechselveränderungen im septischen Schock liefert die Substratutilisation des Herzens: während das gesunde Herz überwiegend Fettsäuren zur Energieproduktion verbrennt, schrumpft deren Anteil im septischen Schock auf $12 \%$. Was aber noch mehr überrascht und bisher unverstanden und auch noch nicht bekannt ist: $40 \%$ seiner Energie schöpft das Herz im septischen Schock aus einem Nährstoff, der keinem der üblichen exogenen Substrate zugeordnet werden kann, sondern aus dem Herzen selbst stammen muß, wobei die sehr geringe Glykogenreserve des Myokards hierfür als Erklärung sicherlich nicht ausreicht (Dhainaut et al. 1993).

Diese ausgeprägten Stoffwechselveränderungen im septischen Schock lassen sich natürlich nicht allein durch $\mathrm{O}_{2}$-Mangel, Ischämie und Energieverarmung erklären. Die sperifische Induktion bestimmter Isoformen 
des Glukosecarrier (GLUT1) durch Tumornekrosefaktor $\alpha$ und Interleukin 1 , die beschriebene Hemmung mitochondrialer Atmungsenzyme durch Stickoxid und die Inhibition des Pyruvatdehydrogenasekomplexes durch den Tumornekrosefaktor $\alpha$ sind einige Beispiele eines sich langsam mehrenden Verständnisses der Beeinflussung des Stoffwechsels durch Schock- und Sepsismediatoren.

Apoptose - der gerichtete Zelltod - findet sich bei Patienten mit Sepsis v.a. in Lymphozyten und intestinalen Epithelzellen, nicht dagegen in nennenswertem Maße im Herzen und anderen parenchymatösen Organen; bei nichtseptischen Schockzuständen ist die Apoptose ein noch selteneres Phänomen wie bei der Sepsis (Hotchkiss et al. 1999; Moldawer 1999).

\section{8}

\section{Zelluläre Mechanismen der akuten septischen} Kardiomyopathie

Zahlreiche der im Schock und in der Sepsis gebildeten Mediatoren (Übersicht und Literaturangaben in MüllerWerdan et al. 1996; Müller-Werdan u. Werdan 1999) können eine Myokarddepression hervorrufen und damit zum Bild der akuten septischen Kardiomyopathie beitragen. Welche von den zahlreichen beschriebenen Wirkungen auf die Herzmuskelzelle jedoch tatsächlich klinische Relevanz besitzen, ist bisher nur mit Einschränkung zu beantworten.

Die derzeit am meisten favorisierte "negativ-inotrope Kaskade“ gibt Abb. 33-11 wieder: Zirkulierendes Endotoxin stimuliert Mediatorzellen zur systemischen und myokardialen Freisetzung von Tumornekrosefaktor $\alpha$ (TNF- $\alpha$ ) und Interleukin 1 (IL-1). TNF- $\alpha$ und (wohl von geringerer Bedeutung) IL-1 induzieren dann die Bildung der induzierbaren Stickoxidsynthase (iNOS) im Herzen; das von ihr gebildete Stickoxid (NO) stimuliert die lösliche Guanylatcyclase des Kardiomyozyten, und der dar-

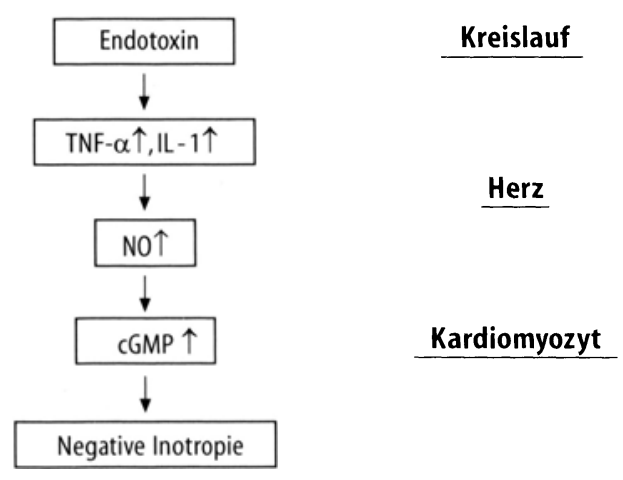

Kardiodepression ausreichend erklärt ??

Abb. 33-11. Kardiodepressive Faktoren in der sepsis - die am besten abgesicherte Kaskade. TNF- $\alpha$ 'Tumornekrosefaktor $\alpha$; II. Interleukin; c(iMP' zyklisches (iuanosinmonophosphat aus resultierende Anstieg des zyklischen Guanosinmonophosphats führt schließlich über eine Hemmung des $\mathrm{Ca}^{2+}$-Einstroms in die Zelle oder über eine Desensibilisierung der Myofilamente gegenüber $\mathrm{Ca}^{2+}$ zur Kardiodepression (Kumar u. Parrillo 1995b).

Alle Einzelschritte sind weitgehend belegt. Die Gabe von Endotoxin führt bei Probanden zur hyperdynamischen Herz-Kreislauf-Situation mit Blutdrucksenkung, Vasodilatation und Steigerung des Herzindex bei gleichzeitiger Abnahme des Schlagarbeitsindex als klinischem Inotropieparameter (Suffredini et al. 1989). Auch der Anstieg des zirkulierenden TNF- $\alpha$ nach Endotoxinapplikation ist bei Probanden gezeigt worden (Michie et al. 1988), und erhöhte TNF- $\alpha$-Plasmaspiegel finden sich auch im septischen Schock. Bei therapeutisch mit TNF behandelten Tumorpatienten kann es zur ausgeprägten Myokarddepression kommen. Im Tierexperiment führt die Gabe von TNF- $\alpha$ zu einer bis mehrere Tage anhaltenden Myokarddepression.

Der Nachweis der direkten depressorischen Wirkung des TNF- $\alpha$ auf die Funktion des Kardiomyozyten läßt sich im Zellkulturexperiment führen (Abb.33-12): TNF- $\alpha$ hemmt in klinisch relevanten Konzentrationen nach mehrstündiger Einwirkungsdauer die „positiv-inotrope“ Wirkung des $\beta$-Adrenozeptoragonisten Isoproterenol und auch die hoher $\mathrm{Ca}^{2+}$-Konzentrationen; die depressorische TNF- $\alpha$-Wirkung kann durch Anti-TNF- $\alpha$ Antikörper neutralisiert werden. Auch die Induktion einer induzierbaren Stickoxidsynthase in Kardiomyozyten durch TNF- $\alpha$ und IL-1 ist experimentell gut belegt, ebenso wie bei Patienten mit septischem Schock eine erhöhte Aktivität der iNOS und ein cGMP-Anstieg im Herzen.

Es spricht also vieles dafür, daß die in Abb. 33-11 gezeigte „negativ-inotrope“ Kaskade für die Myokarddepression in der Sepsis und im septischen Schock wesentlich mitverantwortlich ist. Dennoch scheint dies nicht der einzige kardiodepressive Mechanismus zu sein: einerseits kann TNF- $\alpha$ im Experiment auch stickoxidunabhängig negativ-inotrop wirken (Müller-Werdan et al. 1996), andererseits wird durch TNF- $\alpha$ nicht nur die positiv-inotrope Wirkung von $\beta$-Adrenozeptoragonisten, sondern auch die von $\alpha$-Adrenozeptoragonisten gehemmt (Müller-Werdan et al. 1996), wobei letzteres über eine alleinige Induktion der Stickoxidsynthase nicht $\mathrm{zu}$ erklären ist. So hemmt Endotoxin, das in Kardiomyozyten die Stickoxidsynthase stimuliert, zwar auch die positiv-inotrope Wirkung $\beta$-Adrenozeptoragonisten, nicht aber die von $\alpha$-Adrenozeptoragonisten (Müller-Werdan et al. 1996).

Aufbauend auf experimentellen Befunden dieser Art kann davon ausgegangen werden, daß neben der Stickoxid-cGMP-Kaskade auch noch andere Mechanismen für die negativ-inotrope Wirkung von TNF- $\alpha$ verantwortlich sein müssen. Die belegte Hemmung des Phosphoinositolstoffwechsels durch dieses Zytokin erklärt die Hemmung der positiv-inotropen Wirkung von o-Ad- 
Abb.33-12. Kardiodepression in der Sepsis - Resultat der Beeinträchtigung mehrerer inotroper Signaltransduktionswege. $I L-1$ Interleukin 1 ; TNF- $\alpha$ Tumornekrosefaktor $\alpha$; $C D F$ kardiodepressiver Faktor; NO Stickoxid; $c G M P$ zyklisches Guanosinmonophosphat; $I P_{3}$ Inositoltriphosphat. Weitere Erläuterungen siehe Text und Müller-Werdan u. Werdan 1999 einschließlich Referenzen. (Nach Müller-Werdan u. Werdan 1999)

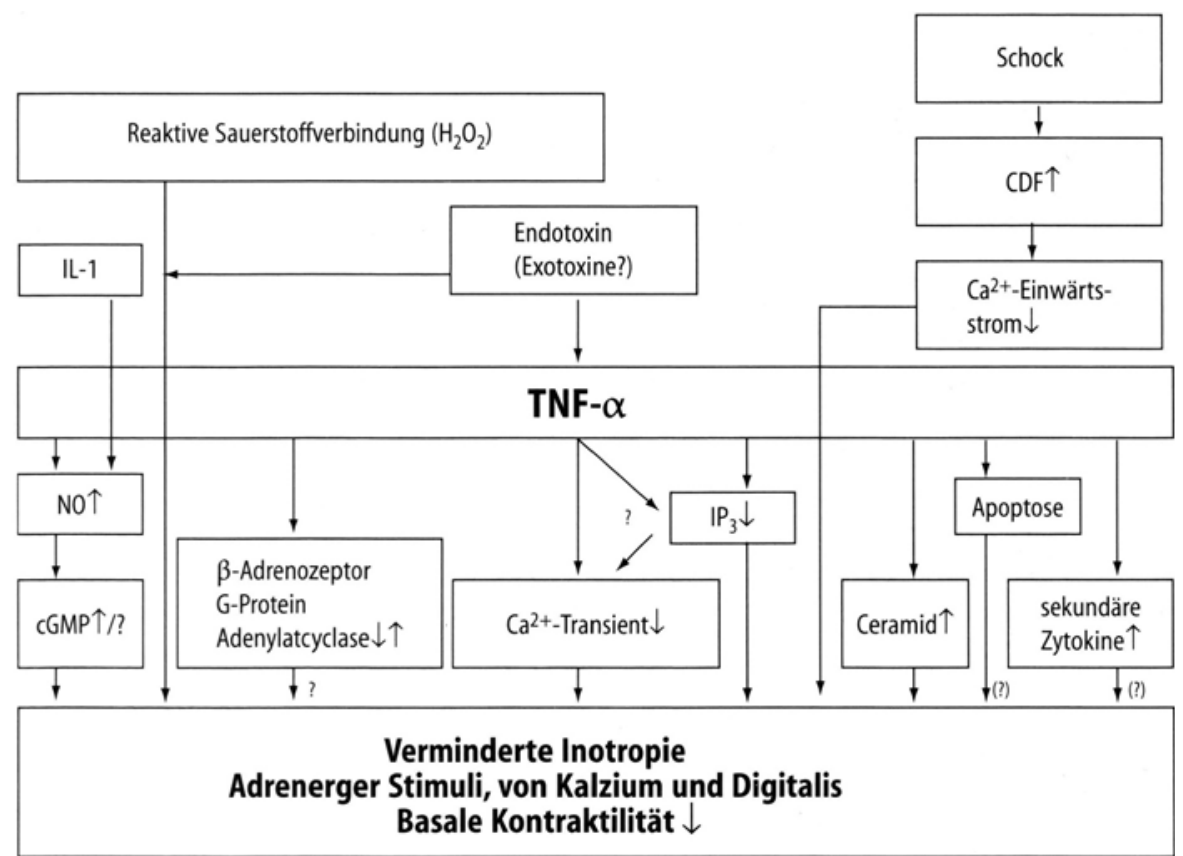

renozeptoragonisten, und die ebenfalls dokumentierte Abschwächung des $\mathrm{Ca}^{2+}$-Transients durch TNF schwächt alle inotropen Effekte ab. Ebenfalls belegt ist die Hemmung des $\mathrm{Ca}^{2+}$-Einwärtsstroms durch einen kardiodepressiven Peptidfaktor, der sich in Hämofiltraten von Patienten mit kardiogenem und septischem Schock in Konzentrationen nachweisen läßt, die diese Hemmwirkung in humanen Herzmuskelzellen hervorrufen (Abb. 33-12; Hallström et al. 1993).

\section{9}

\section{Pathologische Sauerstoffabhängigkeit}

Das Konzept der supranormalen $\mathrm{O}_{2}$-Versorgung von $\mathrm{Pa}$ tienten mit ARDS („adult/acute respiratory distress syndrome"), Sepsis, Schock sowie anderen kritisch Kranken wird seit mehr als 20 Jahren propagiert und praktiziert (Übersicht in Schuster u. Müller-Werdan 2000). Es beruht auf der Annahme einer pathologischen Abhängigkeit des $\mathrm{O}_{2}$-Verbrauchs von der $\mathrm{O}_{2}$-Aufnahme, d.h. der Verschiebung der $\mathrm{O}_{2}$-Aufnahmeschwelle zu höheren Werten. Als Ursache dieser Schwellenverschiebung wird eine Einschränkung der $\mathrm{O}_{2}$-Extraktionsfähigkeit infolge der gestörten Mikrozirkulation angesehen. Als therapeutische Konsequenz ist zu fordern, diesen Patienten ein supranormales $\mathrm{O}_{2}$-Angebot, im wesentlichen durch Steigerung des Herzzeitvolumens, zu ermöglichen.

Ein aktualisiertes Konzept der pathologischen $\mathrm{O}_{2}$ Abhängigkeit kritisch Kranker muß jedoch neben der eingeschränkten $\left(_{2}\right.$-Versorgung infolge der gestörten Mikrozirkulation auch die gehemmte zelluläre (), -Verwertung infolge der mediatorbedingten Inhibition der
Mitochondrienfunktion mit berücksichtigen (Abb.33-3). Die beiden letzten großen klinischen Studien (Abschn. 35.7.3) liefern überzeugende Argumente, daß eine Erhöhung des $\mathrm{O}_{2}$-Angebots allein nicht ausreicht.

\subsection{0}

\section{Reperfusionsschaden}

Die Gewebeschäden sind nicht allein durch den Mangel an Sauerstoff während der Ischämiephase (Downey et al. 1995), sondern auch durch die Reperfusion und Reoxygenation der ischämischen Organe bedingt (Livingston et al. 1993; Mullane 1991; Hearse u. Bolli 1991). Bei der enzymatischen Reaktion der in der Ischämiephase akkumulierten ATP-Abbauprodukte Hypoxanthin und Xanthin mit Sauerstoff kommt es zur Bildung freier $\mathrm{O}_{2}$-Radikale (Hydroperoxid, Superoxidradikal, Hydroxidradikal), welche durch Lipidperoxidation die Aktivierung des Prostaglandinsystems und eine Denaturierung von Zellmembranproteinen bewirken (Abb. 33-9 und 33-10).

Katalysiert wird diese Radikalproduktion durch das Enzym Xanthinoxidase, welches in der Ischämie durch Umwandlung aus der Xanthindehydrogenase (nicht zur Radikalbildung befähigt) entsteht. Dieser Umwandlungsprozeß läuft in den Geweben bei Ischämie unterschiedlich schnell ab (Darm: $10 \mathrm{~s}$; Herzmuskel: 8 min; Leber, Milz, Niere, Lunge: $40 \mathrm{~min}$ ), was die unterschiedliche Empfindlichkeit der einzelnen Gewebe auf Reperfusionsschäden mit erklären soll (McCord 1985). Freie $\mathrm{O}_{2}$-Radikale rufen darüber hinaus eine verstärkte Akkumulation polymorphkerniger leukozyten hervor, begünstigen das leukozytenst icking sowie über den „respiratory burst" die Bildung weiterer ( $)_{2}$-Radikale aus 
polymorphkernigen Leukozyten (Abb. 33-9; Mullane 1991; Smith et al. 1991; Downey et al. 1995).

Da natürliche Schutzstoffe gegen die Effekte der $\mathrm{O}_{2}$-Radikale (Scavengersubstanzen, Superoxiddismutase, Glutathion, Peroxidase, Katalase) im Intrazellulärraum nur in geringer Konzentration vorliegen, können sie den als Folge der Reperfusion und Reoxygenation eintretenden Reperfusionsschaden, der durch Endothelläsion, intrazelluläres Ödem und letztlich deletären Einstrom von Kalzium in die Zellen charakterisiert ist, nicht verhindern. Der Reperfusionsschaden wird deshalb, allerdings nicht unumstritten (Hearse u. Bolli 1991), als wichtiger pathogenetischer Faktor bei der Entwicklung des Multiorgandysfunktionssyndroms (MODS) im Schock gesehen. Der Nachweis der klinischen Wirksamkeit einer Antioxidanzientherapie im Schock steht allerdings noch aus.

33.11

\section{Neurohumorale und para-/autokine Adaptation}

Zur Kompensation der schockbedingten Kreislaufveränderungen stehen dem Organismus verschiedene Sensoren des kardiovaskulären Systems zur Verfügung, die ihre Information zentralnervös gesteuert an die Regelsysteme des Sympathikus und des Endokriniums weiterleiten (Abb. 33-13; Guiterrez u. Brown 1993; Beerthuizen 1993; Bond 1993; Kumar u. Parrillo 1995a; Schmidt et al. 1999). Die einzelnen Sensoren und Regelsysteme werden dabei in unterschiedlichen Schockphasen aktiv.

In der Präschockphase (Tachykardie, Oligurie; noch keine Hypotonie, getrübtes Sensorium, Azidose) werden v.a. die Dehnungsrezeptoren des Niederdrucksystems (rechter Vorhof, Pulmonalarterien) aktiviert, so daß bei einer Abnahme des Intravasalvolumens oder einer $\mathrm{Zu}$ nahme der venösen Kapazität eine Sympathikusaktivierung des medullären Vasomotorenzentrums resultiert. Auch die Barorezeptoren des Aortenbogens, des Karotissinus und der Splanchnikusgefäße zeigen bereits einen geringen Blutdruckabfall an und aktivieren damit ebenso Sympathikusfasern des medullären Vasomotorenzentrums.

Sinkt der mittlere Blutdruck auf Werte unter 80-90 $\mathrm{mmHg}$, so kommt die Aktivität der Barorezeptoren des Aortenbogens zum Erliegen, ebenso die des Karotissinus bei Druckwerten unter $60 \mathrm{mmHg}$. Ab diesem Bereich tragen die vaskulären Chemorezeptoren, die unter physiologischen Bedingungen eher im Hintergrund stehen, entscheidend zur zentralen Sympathikusaktivierung bei: Die Abnahme des $\mathrm{pO}_{2}$, die Zunahme des $\mathrm{pCO}_{2}$ und der Abfall des $\mathrm{pH}$-Werts sind ihre Aktivatoren. Der stärkste Stimulus für die Sympathikusaktivierung des medullären Vasomotorenzentrums sind jedoch bei mittleren Blutdruckwerten unter $60 \mathrm{mmHg}$ die medullären Chemorezeptoren: Sie werden bei einer Zunahme des pC(O, in Kombination mit einer zerebralen Ischämie aktiv und stimulieren das Herz-Kreislauf-System maximal im
Sinne der Aufrechterhaltung eines ausreichenden Blutdrucks.

Auch das antidiuretische Hormon (ADH, Vasopressin) spielt hierbei eine entscheidende Rolle. Während die ADH-Freisetzung aus der Hypophyse unter physiologischen Bedingungen der Regulation hypothalamischer Kerne auf Änderungen der Serumosmolarität folgt, dominiert bei extensiver Hypovolämie die Stimulation von Dehnungsrezeptoren die ADH-Freisetzung und führt damit zur Wasserretention. Der juxtaglomeruläre Apparat der Niere reagiert auf seine Minderperfusion bei Hypovolämie und Schock mit einer Freisetzung von Renin.

Die Kompensationsmechanismen im Schock dienen dazu, den $\mathrm{O}_{2}$-Transport zu lebenswichtigen Organen sicherzustellen. Sie laufen bei verschiedenen Schockstadien und Schockformen in qualitativ ähnlicher Weise, quantitativ aber unterschiedlichem Ausmaß ab und lassen sich jeweils einer der 4 Kategorien zuordnen: - Aufrechterhaltung des venösen Zirkulationsdrucks,

- Optimierung der Herzfunktion,

- Blutumverteilung zur Aufrechterhaltung der adäquaten Durchblutung der Vitalorgane,

- Erleichterung der $\mathrm{O}_{2}$-Abgabe im Gewebe.

\subsection{1 \\ Aufrechterhaltung des venösen Zirkulationsdrucks}

Hierzu dient zum einen die Auffüllung des Intravasalvolumens durch Rückverteilung von Flüssigkeit aus dem Interstitium und dem Intrazellulärraum in die Blutgefäße und die Einschränkung der Nierenausscheidung über eine Abnahme der glomerulären Filtrationsrate und über einen Anstieg von Aldosteron und ADH; zum anderen läßt sich der Zirkulationsdruck auch durch eine Zunahme des Venentonus (Abnahme der venösen Kapazität; s. Abb. 33-2) erzielen, und zwar durch die Steigerung der Sympathikusaktivität und des zirkulierenden Adrenalins, Angiotensins und antidiuretischen Hormons.

Die Rückverteilung von Flüssigkeit aus dem Interstitium in den Intravasalraum ist ein rasch einsetzendes Phänomen. Es basiert auf der Abnahme des hydrostatischen Kapillardrucks infolge der sympathikusvermittelten präkapillären Vasokonstriktion. Der erzielte transkapilläre hydrostatisch-osmotische Druckgradient treibt den transkapillären Plasmaeinstrom, der einen Blutverlust von 500-1000 ml innerhalb von $24-48$ h vollständig ausgleichen kann. Der Anstieg des onkotischen Plasmadrucks infolge der Schockhyperglykämie unterstützt weiterhin die Auffüllung des Intravasalraums aus dem Interstitium und dem Intrazellulärraum.

Die Einschränkung der Nierenausscheidung kommt weniger in der akuten Schockphase als im weiteren Verlauf zum Tragen. Die Abnahme des Herzindex und die Konstriktion afferenter Arteriolen mindern den Nierenperfusionsdruck und senken so einerseits über die Ein- 


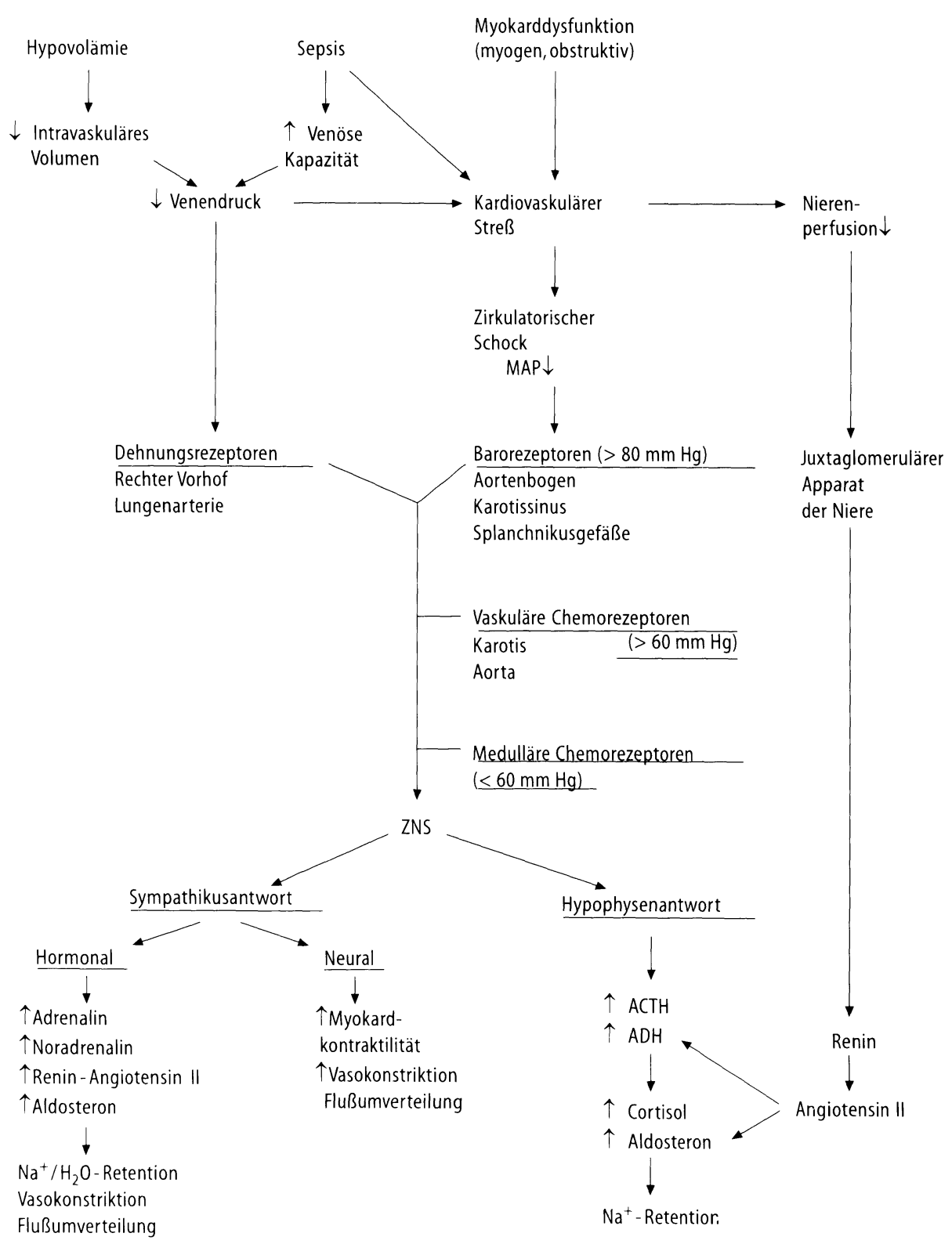

Abb. 33-13. Neurohumorale Schockadaptation. In der frühen kardiovaskulären Streßphase kann die neurohumorale Adaptation auf die gesteigerte Aktivität des juxtaglomerulären Apparates und die Niederdruckmechanorezeptoren des rechten Vorhofs und der Lungenarterien limitiert sein. Im Falle der Schockprogression kommt es zu einer sequentiellen Stimulation der Hochdruckbarorezeptoren, der vaskulären Chemorezeptoren und der medullären Chemorezeptoren. Die Folge davon ist eine verstärkte neurohumorale Ak-

tivität mit Steigerung der Hypophysensekretion von ACTH und $\mathrm{ADH}$ und einer intensiven Sympathikusstimulation durch das medulläre Kreislaufzentrum mit dem Ziel einer Volumenretention, einer Anhebung des Venentonus, einer Steigerung der Herzfunktion und einer Blutflußumverteilung zugunsten der Vitalorgane. MAP mittlerer arterieller Blutdruck; ZNS Zentralnervensystem; $A C T H$ adrenokortikotropes Hormon; $A D H$ antidiuretisches Hormon. (Mod. nach Kumar u. Parrillo 1995a)

schränkung der glomerulären Filtrationsrate das Urinvolumen; andererseits führt die renale Perfusionsminderung zusammen mit der Sympathikusaktivierung und Veränderungen der Tubulusflüssigkeit zur Freisetzung von Renin aus dem juxtaglomerulären Apparat, welches über die Ingangsetzung der Angiotensinogen/ Angiotensin-I/II-Kaskade zur Freisetzung von Aldosteron aus der Nebennierenrinde führt. Angiotensin II

ist ein potenter Vasokonstriktor, besonders im Bereich der Splanchnikusgefäße, und aktiviert den Sympathikus und die Adrenalinfreisetzung aus der Nebenniere; Aldosteron steigert die Rückresorption von $\mathrm{Na}^{+}$im distalen Tubulus im Gegentausch mit $\mathrm{K}^{+}$und $\mathrm{H}^{+}$.

Die bereits beschriebene Freisetzung von ADH aus dem Hypophysenhinterlappen durch Stimulation der Dehnungsrezeptoren im rechten Vorhof wird weiterhin 
durch Angiotensin II über eine Sympathikusaktivierung verstärkt. ADH bewirkt eine renale Wasserrückresorption auf Kosten der Osmolarität und mit der Gefahr einer Hyponatriämie. Außerdem wirkt es vasokonstriktorisch, besonders auf die Splanchnikusgefäße. Zur Stabilisierung des zirkulatorischen Venendrucks trägt auch entscheidend die Venokonstriktion (besonders der venösen Kapazitätsgefäße des Splanchnikuskreislaufs) durch die gesteigerte Sympathikusaktivität sowie durch das zirkulierende Adrenalin, ADH und Angiotensin II bei.

\subsection{2}

\section{Optimierung der Herzfunktion}

Katecholamine (lokal im Myokard aus den Sympathikusnervenendigungen freigesetztes Noradrenalin und zirkulierendes Adrenalin der Nebenniere) besetzen die $\beta_{1}$-Adrenozeptoren des Herzens, wirken so positiv-inotrop und positiv-chronotrop, steigern dadurch das Herzzeitvolumen und stabilisieren auf diese Weise den Blutdruck. Auch die Besetzung der $\beta_{2^{-}}, \alpha_{1}$-Adrenozeptoren mit diesen Katecholaminen trägt in geringerem Maße dazu bei, ebenso wie Angiotensin II durch eine direkte und indirekte (via Sympathikusaktivierung) positiv-inotrope Wirkung. Die gesteigerte Herzfunktion hat auch eine Senkung des rechtsatrialen Drucks zur Folge, was wiederum den venösen Rückstrom erhöht (s. Abschn. 33.1).

\subsection{3}

\section{Blutumverteilung}

Die erhöhte Sympathikusaktivität und die systemische Freisetzung von Adrenalin, ADH und Angiotensin II im
Schock bewirken in allen sensitiven Gefäßbetten (Haut, Skelettmuskel, Nieren, Splanchnikusorgane) eine Vasokonstriktion. Damit wird eine Umverteilung des Blutflusses $\mathrm{zu}$ den autoregulierten Vitalorganen Hirn und Herz erzielt (Tabelle 33-1).

\subsection{4}

\section{Erleichterung der Sauerstoffabgabe im Gewebe}

Die lokale Azidose in mangelperfundiertem Gewebe vermindert die Affinität des Hämoglobins zu Sauerstoff (Rechtsverschiebung der $\mathrm{O}_{2}$-Dissoziationskurve) und erleichtert somit die $\mathrm{O}_{2}$-Abgabe ebenso wie die Zunahme des $\mathrm{O}_{2}$-Diffusionsgradienten im $\mathrm{O}_{2}$-verarmten $\mathrm{Ge}$ webe. Eine respiratorische Alkalose im zirkulatorischen Schock steigert die Synthese des 2,3-Diphosphoglycerats (DPG) in den Erythrozyten, was ebenfalls eine Rechtsverschiebung der $\mathrm{O}_{2}$-Dissoziationskurve bewirkt. Mit zunehmender Laktatazidose fallen die 2,3-DPG-Spiegel, aber die zunehmende Azidose kompensiert diesen Effekt bezüglich der $\mathrm{O}_{2}$-Abgabe. Hypothermie führt zu einer Linksverschiebung der $\mathrm{O}_{2}$-Dissoziationskurve; bei der Schockerstversorgung sollte deshalb eine Hypothermie verhindert werden.

In diese neurohumorale Schockadaptation greifen wir mit unseren therapeutischen Maßnahmen durch qualitativ unterschiedliche Ansätze teils verstärkend, teils abschwächend, teils modifizierend ein. Para- und autokrine schockinduzierte Mechanismen, wie z.B. die exzessive Stickoxidproduktion in den Gefäßen durch Induktion der Stickoxidsynthase, können diese Adaptationsmechanismen jedoch antagonisieren und den labilen Schockzustand zur Dekompensation bringen. 